\title{
Barrier estimates for a critical Galton-Watson process and the cover time of the binary tree
}

\author{
David Belius, Jay Rosen*, Ofer Zeitouni ${ }^{\dagger}$ \\ February 13, 2017
}

\begin{abstract}
For the critical Galton-Watson process with geometric offspring distributions we provide sharp barrier estimates for barriers which are (small) perturbations of linear barriers. These are useful in analyzing the cover time of finite graphs in the critical regime by random walk, and the Brownian cover times of compact two dimensional manifolds. As an application of the barrier estimates, we prove that if $C_{L}$ denotes the cover time of the binary tree of depth $L$ by simple walk, then $\sqrt{C_{L} / 2^{L+1}}-$ $\sqrt{2 \log 2} L+\log L / \sqrt{2 \log 2}$ is tight. The latter improves results of Aldous (1991), Bramson and Zeitouni (2009) and Ding and Zeitouni (2012). In a subsequent article we use these barrier estimates to prove tightness of the Brownian cover time for the two-dimensional sphere.
\end{abstract}

\section{Introduction and statement of main results}

Let $P_{n}$ be the law of the critical Galton-Watson process $\left(T_{l}\right)_{l \geq 0}$ with inital population $T_{0}=n$ and geometric offspring distribution. For any $a, b, L$ let

$$
f_{a, b}(l ; L)=a+(b-a) \frac{l}{L},
$$

be the line interpolating $a$ and $b$ over the interval $[0, L]$. Abbreviate $l_{L}=$ $l \wedge(L-l)$. Let $\mathbb{N}=\{1,2, \ldots\}$ and $\mathbb{Z}^{+}=\{0,1,2, \ldots\}$. For $y, \delta \geq 0$, let $H_{y, \delta}=[y, y+\delta]$ and set $H_{y}=H_{y, 1}$. The main result of this article is the following barrier estimates for the process $T_{l}$.

\footnotetext{
* Partially supported by grants from the NSF and from the Simons foundation

${ }^{\dagger}$ Partially supported by the ERC advanced grant LogCorrelatedFields
} 


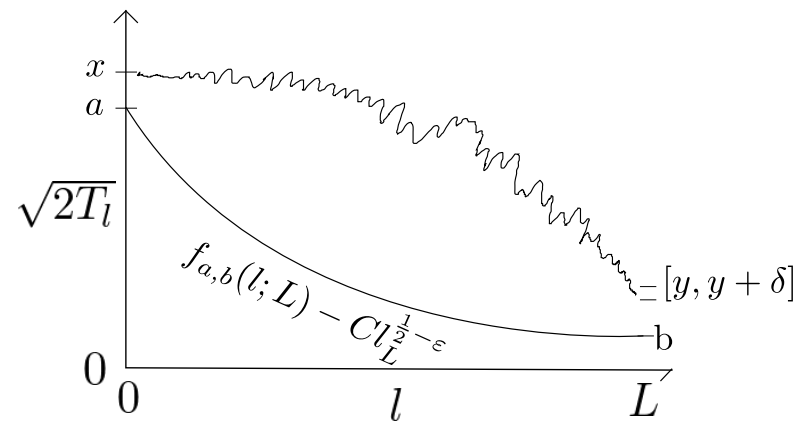

Figure 1: Illustration of barrier event in Theorem 1.1 a).

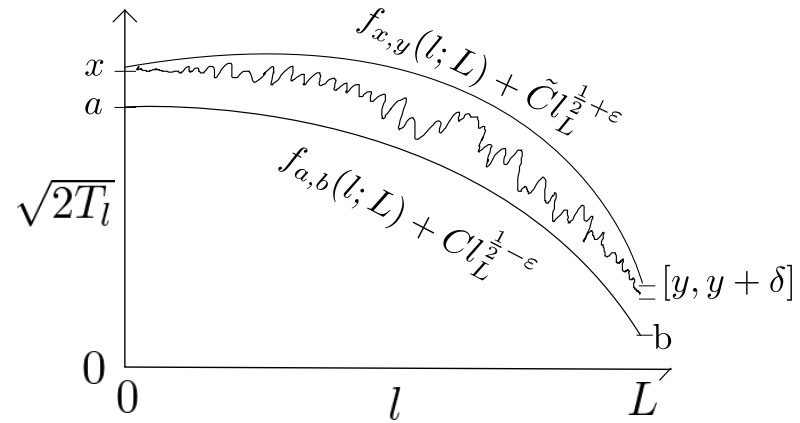

Figure 2: Illustration of barrier event in Theorem 1.1 b). 
Theorem 1.1. a) For all fixed $\delta, C>0, \eta>1$ and $\varepsilon \in\left(0, \frac{1}{2}\right)$ we have, uniformly in $\sqrt{2} \leq x, y \leq \eta L$ such that $x^{2} / 2 \in \mathbb{N}$, any $0 \leq a \leq x, 0 \leq b \leq y$, that

$$
\begin{aligned}
& P_{x^{2} / 2}\left(f_{a, b}(l ; L)-C l_{L}^{\frac{1}{2}-\varepsilon} \leq \sqrt{2 T_{l}}, l=1, \ldots, L-1, \sqrt{2 T_{L}} \in H_{y, \delta}\right) \\
& \leq c \frac{(1+x-a)(1+y-b)}{L} \sqrt{\frac{x}{y L}} e^{-\frac{(x-y)^{2}}{2 L}} .
\end{aligned}
$$

If in addition $a \geq L / \eta$, similar bounds hold for $y \leq \sqrt{2}$, with $\sqrt{\frac{x}{y L}}$ replaced by 1.

b) For any $\tilde{C} \geq 2 C+2 \delta+\eta+\sqrt{2}$, if, in addition to the conditions in part a), we also have $(1+x-a)(1+y-b) \leq \eta L, \max (a b,|a-b|) \geq L / \eta$ and $[y, y+\delta] \cap \sqrt{2 \mathbb{Z}^{+}} \neq \emptyset$ then

$$
\begin{aligned}
& P_{x^{2} / 2}\left(f_{a, b}(l ; L)+C l_{L}^{\frac{1}{2}-\varepsilon} \leq \sqrt{2 T_{l}} \leq f_{x, y}(l ; L)+\tilde{C} l_{L}^{\frac{1}{2}+\varepsilon}, l=\underset{\sqrt{2 T_{L}} \in H_{y, \delta}}{1, \ldots, L-1,}\right) \\
& \geq c \frac{(1+x-a)(1+y-b)}{L} \times\left(\sqrt{\frac{x}{y L}} \wedge 1\right) e^{-\frac{(x-y)^{2}}{2 L}},
\end{aligned}
$$

and the estimate is uniform in such $x, y, a, b$ and all $L$.

Remark 1.2. a) In this paper constants, whose value may change from occurence to occurence, that depend at most on $C, \delta, \eta$ and $\varepsilon$, are denoted by $c$. The notation $a \asymp b$ means that $a \leq c \cdot b$ and $b \leq c \cdot a$.

b) When $\delta<\sqrt{2}$ and $y=0$ the terminal condition $\sqrt{2 T_{L}} \in y \in H_{y, \delta}$ is equivalent to $T_{L}=0$. Less precise barrier estimates for the process $T_{l}$ conditioned on $T_{L}=0$ appear in [6, Proposition 7.1].

We will put Theorem 1.1 in the context of estimates for Bessel processes, see Proposition 1.4 below. Before doing so, we emphasize that the main application of Theorem 1.1 is in an upcoming paper by the authors which proves tightness of the (centered) square-root of the Brownian cover time of the two dimensional sphere. In the current paper, we illustrate the use of Theorem 1.1 by presenting a quick proof of a similar result for the cover time of the binary tree. Let $\mathcal{T}_{L}$ be the tree of depth $L$, with a root of degree one attached to the top. (Formally, begin with a binary tree rooted at vertex $o$, and attach to it a vertex $\rho$, the root of $\mathcal{T}_{L}$, connected by an edge to $o$.) The tree $\mathcal{T}_{L}$ has $2^{L+1}$ vertices and $2^{L}+1$ leaves (including $\rho$ ). Let $\mathbb{P}$ be the law of discrete time simple random walk $\left(X_{n}\right)_{n \geq 0}$ on $\mathcal{T}_{L}$ starting at the root. Let $\tau_{y}, y \in \mathcal{T}_{L}$, be 
the hitting time of the vertex $y$ by the random walk. The cover time

$$
C_{L}=\max _{y \in \mathcal{T}_{L}} \tau_{y}=\max _{y \in \mathcal{T}_{L}, y \text { is a leaf }} \tau_{y}
$$

is the first time the random walk has visited every vertex of $\mathcal{T}_{L}$. We prove the following estimate.

Theorem 1.3. There exist constants $c$ such that for all $x>0$,

$$
\begin{gathered}
\limsup _{L \rightarrow \infty} \mathbb{P}\left(\sqrt{\frac{C_{L}}{2^{L+1}}} \geq \sqrt{2 \log 2} L-\frac{1}{\sqrt{2 \log 2}} \log L+x\right) \leq c x e^{-x \sqrt{2 \log 2}}, \\
\liminf _{L \rightarrow \infty}\left(\sqrt{\frac{C_{L}}{2^{L+1}}} \geq \sqrt{2 \log 2} L-\frac{1}{\sqrt{2 \log 2}} \log L+x\right) \geq c x e^{-x \sqrt{2 \log 2}}
\end{gathered}
$$

and

$$
\limsup _{L \rightarrow \infty} \mathbb{P}\left(\sqrt{\frac{C_{L}}{2^{L+1}}} \leq \sqrt{2 \log 2} L-\frac{1}{\sqrt{2 \log 2}} \log L-x\right) \leq e^{-c x} .
$$

In particular, Theorem 1.3 shows that

$$
\sqrt{\frac{C_{L}}{2^{L+1}}}=\sqrt{2 \log 2} L-\frac{1}{\sqrt{2 \log 2}} \log L+O(1)
$$

that is, tightness of a centered, scaled version of the square root of the cover time. Equivalently one could state tightness directly in terms of a centered and scaled version of the cover time itself, as

$$
\frac{C_{L}}{2^{L+1} L}=2(\log 2 \cdot L-\log L+O(1))
$$

and the corresponding tail bounds can also be written in a similar way. The statement 1.5 improves on the estimate from [14], which has $O\left((\log \log L)^{8}\right)$ in place of $O(1)$. (Earlier results of Aldous [4] give the leading order $\sqrt{2 \log 2} L(1+o(1))$.) Theorem 1.3 also provides a new proof that after appropriate centering, $\sqrt{C_{L} / 2^{L+1}}$ is tight, a result proven in [9] using a recursion (in a way that avoids computing the centering term).

In light of recent works [14, 6], it appears that cover times of homogeneous trees and of two dimensional graphs or manifolds are related to the extrema of certain critical hierarchical random fields, a universality class which contains 
logarithmically correlated fields and branching random walks ${ }^{1}$. The logarithmic correction term in (1.5), whose form was proven in 14, is, up to constant multiple, the universal correction term ${ }^{2}$ for fields in this universality class. That the minimum (or maximum) is tight after centering by the leading term together with this logarithmic correction term is another conjectured universal feature of these fields, as is the decay $x e^{-c x}$, for some $c$, of the right tail, which we verify in (1.2) and (1.3). (By contrast, the left tail for which we have only the rough bound (1.4), is not expected to be universal, and in general is not even of exponential form.) Our approach builds on previous works on branching random walk [7, [2], [5], 3], 8] and cover times [11, [14, 6]. More precisely, we use a second moment method with a truncation involving the process of discrete edge local times staying above certain barriers. The barrier estimates that are the main results of this paper are a crucial technical input.

The main step of the proof of the cover time result is the analysis of the minimum of the discrete local times among the leaves at the time a certain local time is reached at the root. For the maximum of continuous local times on the leaves more precise results have been obtained [1].

The intuition behind Theorem 1.1 is that the process $l \rightarrow \sqrt{2 T_{l}}$ behaves like a Bessel-0 process, i.e. a process $Y_{t}$ which satisfies the SDE

$$
d Y_{t}=d W_{t}-\frac{1}{2 Y_{t}} d t
$$

for a Brownian motion $W_{t}$ (the drift is only significant if $Y_{t}$ is close to zero, otherwise the process $Y_{t}$ behaves like a Brownian motion). The Galton-Watson process $T_{l}$ can be thought of as a discrete version of a squared Bessel-0 process. Indeed, if $f_{l, j}, j \geq 1$, are the number of offspring of each individual in generation $l$, letting $g_{l, j}=f_{l, j}-1$ we can also write $\sqrt{2 T_{l+1}}$ as

$$
\sqrt{2 T_{l+1}}=\sqrt{2 T_{l}} \sqrt{1+\frac{1}{T_{l}} \sum_{j=1}^{T_{l}} g_{l, j}} .
$$

When $T_{l}$ is large one can Taylor expand the square root to obtain that the

\footnotetext{
${ }^{1}$ More generally, isomorphism theorems have been used to show that for any finite connected graph for which hitting times are asymptotically shorter than cover times, the cover time divided by the number of edges is of the same order as the square of the maximum of the Gaussian Free Field on the same graph [13] for arbitrary trees or for bounded degree graphs, they match to leading order [12].

${ }^{2}$ The constant multiple is determined by tail estimates. Differing tail estimates for local times and related Gaussian fields lead to differing constants for cover times and the maximum of such Gaussian fields, as explained in [6, Section 1.2].
} 
increment $\sqrt{2 T_{l+1}}-\sqrt{2 T_{l}}$ equals

$$
\frac{1}{\sqrt{2 T_{l}}} \sum_{j=1}^{T_{l}} g_{l, j}-\frac{1}{2 \sqrt{2 T_{l}}}
$$

plus terms that can be shown to be negligible. The distribution of the normalized sum in (1.8) will be close to Gaussian by the central limit theorem (the $g_{l, j}$ are independent, have mean zero, and are independent of $T_{l}$ ). Thus if we let $Z_{l}=\sqrt{2 T_{l}}$ we can informally write

$$
Z_{l+1}-Z_{l} \approx N_{l}-\frac{1}{2 Z_{l}}
$$

for approximately Gaussian independent $N_{l}$, making the heuristic link to 1.6 apparent. A proof of results similar to Theorem 1.1, that could be extended to other Galton-Watson processes, could be provided 3 by following these ideas. Instead, in this paper, we use exact equalities in law to provide a shorter proof.

Theorem 1.1 should be understood in the light of the following precise large deviation estimate for $\sqrt{2 T_{L}}$.

Proposition 1.4. For all fixed $\delta>0$ and $\eta>1$ we have uniformly in $\sqrt{2} \leq$ $x, y \leq \eta L$ such that $x^{2} / 2$ is an integer that

$$
P_{x^{2} / 2}\left(\sqrt{2 T_{L}} \in H_{y, \delta}\right) \leq c \sqrt{\frac{x}{y L}} e^{-\frac{(x-y)^{2}}{2 L}} .
$$

If, in addition, $[y, y+\delta] \cap \sqrt{2 \mathbb{Z}^{+}} \neq \emptyset$ and $L / \eta \leq x y$, then the corresponding lower bound

$$
P_{x^{2} / 2}\left(\sqrt{2 T_{L}} \in H_{y, \delta}\right) \geq c \sqrt{\frac{x}{y L}} e^{-\frac{(x-y)^{2}}{2 L}},
$$

also holds. Also, for any $0<x \leq \eta L$,

$$
P_{x^{2} / 2}\left(\sqrt{2 T_{L}}=0\right) \asymp e^{-\frac{x^{2}}{2 L}} .
$$

The bounds (1.9)-(1.11) are the same as the ones satisfied by $Y_{L}$ if the process $Y_{t}$, under $P_{x}^{Y}$, is a Bessel process of dimension zero, since

$$
P_{x}^{Y}\left(Y_{L} \in \cdot\right)=\delta_{0} e^{-\frac{x^{2}}{2 L}}+1_{(0, \infty)} \frac{x}{L} e^{-\frac{x^{2}+y^{2}}{2 L}} I_{1}\left(\frac{x y}{L}\right) d y,
$$

\footnotetext{
${ }^{3}$ and is available from the authors
} 
see above Lemma 2.5. Furthermore e.g. Theorem 1.1 a) can be written as

$$
\begin{aligned}
& P_{x^{2} / 2}\left(f_{a, b}(l ; L)-C l_{L}^{\frac{1}{2}-\varepsilon} \leq \sqrt{2 T_{l}}, l=1, \ldots, L-1 \mid \sqrt{2 T_{L}} \in H_{y, \delta}\right) \\
& \leq c \frac{(1+x-a)(1+y-b)}{L} .
\end{aligned}
$$

The probability that a Brownian bridge in the time interval $[0, L]$ stays above a linear barrier (or a small perturbation thereof) during $[1, L-1]$ as in the event in (1.13), when it starts at distance $x-a$ from the line and ends at distance $y-b$ from it is of the order of the right-hand side of 11.13 ) (see 2.2 and $(2.3)$ below). Our Theorem 1.1 can thus be thought of as a Galton-Watson process version of barrier results for the Brownian bridge. Heuristically, it arises from approximating $\sqrt{2 T_{l}}$ conditioned on its end point by a Brownian bridge.

In the rest of the paper, we prove Theorems 1.1 and 1.3 . We begin by proving, in Section 2, barrier estimates for 0-dimensional Bessel processes. In Section 3, we show that traversal counts and local times of a random walk on $\mathbb{Z}^{+}$give a Markovian structure closely related to the sampling of a Bessel-0 process. In Section 4, we use the latter structure to transfer barrier estimates for Bessel-0 processes to the setting of Theorem 1.1. Finally, in Section 5 we use a first/second moment method, together with the barrier estimates of Theorem 1.1, to obtain Theorem 1.3 .

\section{Barrier estimates for the 0-dimensional Bessel process}

We will derive the barrier estimate in Theorem 1.1 from similar results for a Brownian motion. Let $P_{x}^{W}$ be the law of a Brownian motion $W_{t}, t \geq 0$, starting at $x$, and let $P_{x}^{W}\left(\cdot \mid W_{L}=y\right)$ be the law of a Brownian bridge starting at $x$ and ending at $y$ at time $L$. The probability that a Brownian bridge stays above a linear barrier is explicit: If $a \leq x$ and $b \leq y$ then by the reflection principle, see e.g. [7, Lemma 2.2],

$$
P_{x}^{W}\left(W_{l} \geq f_{a, b}(l ; L), l \in[0, L] \mid W_{L}=y\right)=1-\exp \left(-2 \frac{(x-a)(y-b)}{L}\right) .
$$

If also $(x-a)(y-b)=O(L)$ this implies

$$
P_{x}^{W}\left(W_{l} \geq f_{a, b}(l ; L), l \in[0, L] \mid W_{L}=y\right) \asymp \frac{(x-a)(y-b)}{L} .
$$


and by conditioning on $W_{1}$ and $W_{L-1}$ one easily derives that

$$
P_{x}^{W}\left(W_{l} \geq f_{a, b}(l ; L), l \in[1, L-1] \mid W_{L}=y\right) \asymp \frac{(1+x-a)(1+y-b)}{L} .
$$

The next lemma shows that the probability has the same order of magnitude if a "bump" is added or subtracted from the straight line.

Lemma 2.1. For all fixed $\varepsilon \in\left(0, \frac{1}{2}\right), \delta>0, \eta>1, \tilde{C} \geq C+\delta>0$, one has uniformly in $a \leq x, b \leq y,(x-a)(y-b) \leq \eta L,|x-y| \leq \eta L$, and $L$ large enough,

$$
\begin{aligned}
& P_{x}^{W}\left(f_{a, b}(l ; L)+C l_{L}^{\frac{1}{2}-\varepsilon} \leq W_{l} \leq f_{x, y}(l ; L)+\tilde{C} l_{L}^{\frac{1}{2}+\varepsilon}, l \in[1, L-1], W_{L} \in H_{y, \delta}\right) \\
& \asymp P_{x}^{W}\left(f_{a, b}(l ; L)-C l_{L}^{\frac{1}{2}-\varepsilon} \leq W_{l}, l \in[1, L-1], W_{L} \in H_{y, \delta}\right) \\
& \asymp \frac{(1+x-a)(1+y-b)}{L} \frac{1}{\sqrt{L}} e^{-\frac{(x-y)^{2}}{2 L}} .
\end{aligned}
$$

Remark 2.2. The condition $(x-a)(y-b) \leq \eta L$ is not necessary for the upper bounds. One can simply drop the barrier. If we eliminate the condition $|x-y| \leq \eta L$ then the last line in 2.3. would become

$$
\frac{(1+x-a)(1+y-b)}{L} \frac{1}{\sqrt{L}} \sup _{z \in H_{y, \delta}} e^{-\frac{(x-z)^{2}}{2 L}} .
$$

for the upper bound, and inf instead of sup for the lower bound. The condition $|x-y| \leq \eta L$ is only used to guarantee that the supremum in (2.4) has the same order as $e^{-(x-y)^{2} / 2 L}$.

Proof of Lemma 2.1. It follows from Lemma 2.6, Lemma 2.7 and Proposition 6.1 of [7] that for any $C>0$ there is an $r$ large enough so that for all $L>2 r$

$$
\begin{aligned}
& P_{x}^{W}\left(f_{a, b}(l ; L)+C l_{L}^{\frac{1}{2}-\varepsilon} \leq W_{l} \leq f_{x, y^{\prime}}(l ; L)+C l_{L}^{\frac{1}{2}+\varepsilon}, l \in[r, L-r] \mid W_{L}=y^{\prime}\right) \\
& \geq c P_{x}^{W}\left(f_{a, b}(l ; L)-C l_{L}^{\frac{1}{2}-\varepsilon} \leq W_{l}, l \in[r, L-r] \mid W_{L}=y^{\prime}\right) .
\end{aligned}
$$

Let

$$
A=\left\{f_{a, b}(l ; L)+C l_{L}^{\frac{1}{2}-\varepsilon} \leq W_{l} \leq f_{x, y^{\prime}}(l ; L)+C l_{L}^{\frac{1}{2}+\varepsilon}, l \in[1, r] \cup[L-r, L-1]\right\} .
$$


For any fixed $r>0$ and and for all $l \geq 1$ we have

$$
\begin{aligned}
& P_{x}^{W}\left(A \mid f_{a, b}(l ; L)+C l_{L}^{\frac{1}{2}-\varepsilon} \leq W_{l} \leq f_{x, y^{\prime}}(l ; L)+C l_{L}^{\frac{1}{2}+\varepsilon}, l \in[r, L-r], W_{L}=y^{\prime}\right) \\
& \quad \geq c>0
\end{aligned}
$$

for a constant $c$ depending only on $r$ and $C$. Therefore also

$$
\begin{aligned}
& P_{x}^{W}\left(f_{a, b}(l ; L)+C l_{L}^{\frac{1}{2}-\varepsilon} \leq W_{l} \leq f_{x, y^{\prime}}(l ; L)+C l_{L}^{\frac{1}{2}+\varepsilon}, l \in[1, L-1] \mid W_{L}=y^{\prime}\right) \\
& \geq c P_{x}^{W}\left(f_{a, b}(l ; L)-C l_{L}^{\frac{1}{2}-\varepsilon} \leq W_{l}, l \in[1, L-1] \mid W_{L}=y^{\prime}\right) .
\end{aligned}
$$

Since the inequality in the opposite direction, with $c=1$, is trivial, we see by sandwiching that we now have shown

$$
\begin{aligned}
& P_{x}^{W}\left(f_{a, b}(l ; L)+C l_{L}^{\frac{1}{2}-\varepsilon} \leq W_{l} \leq f_{x, y^{\prime}}(l ; L)+C l_{L}^{\frac{1}{2}+\varepsilon}, l \in[1, L-1] \mid W_{L}=y^{\prime}\right) \\
& \asymp P_{x}^{W}\left(f_{a, b}(l ; L) \leq W_{l}, l \in[1, L-1] \mid W_{L}=y^{\prime}\right) \\
& \asymp P_{x}^{W}\left(f_{a, b}(l ; L)-C l_{L}^{\frac{1}{2}-\varepsilon} \leq W_{l}, l \in[1, L-1] \mid W_{L}=y^{\prime}\right) .
\end{aligned}
$$

The claim (2.3) then follows by first using (2.2), then multiplying by the Gaussian density, and then integrating over $y^{\prime} \in H_{y, \delta}$, using the fact that $f_{x, y^{\prime}}(l ; L) \leq f_{x, y+\delta}(l ; L) \leq f_{x, y}(l ; L)+\delta$.

The next lemma shows that we can replace the barrier $f_{a, b}(l ; L)-C l_{L}^{\frac{1}{2}-\varepsilon}$ "checked" for all $l \in[1, L-1]$ with one "checked" only at integer times $l=$ $1, \ldots, L-1$.

Lemma 2.3. For all fixed $C>0, \varepsilon \in\left(0, \frac{1}{2}\right), \delta>0$ and $\eta>1$, one has uniformly in $a \leq x, b \leq y,|x-y| \leq \eta L$ and $L$ large enough,

$$
\begin{aligned}
& P_{x}^{W}\left(f_{a, b}(l ; L)-C l_{L}^{\frac{1}{2}-\varepsilon} \leq W_{l}, l=1, \ldots, L-1, W_{L} \in H_{y, \delta}\right) \\
& \quad \leq c \frac{(1+x-a)(1+y-b)}{L} \frac{1}{\sqrt{L}} e^{-\frac{(x-y)^{2}}{2 L}} .
\end{aligned}
$$

Proof. Let

$$
A_{L}=\left\{f_{a, b}(l ; L)-C l_{L}^{\frac{1}{2}-\varepsilon} \leq W_{l}, l=1, \ldots, L-1\right\}
$$


and for a $\theta>1$ to be fixed later

$$
h_{l}(u, v)=P_{u}^{W}\left(f_{a, b}(l+t ; L)-\theta C(l+t)_{L}^{\frac{1}{2}-\varepsilon} \leq W_{t}, t \in[0,1] \mid W_{1}=v\right) .
$$

Note that $h_{l}(u, v)$ is monotone increasing in $u, v$. Hence using the Markov property we have

$$
\begin{aligned}
& P_{x}^{W}\left(f_{a, b}(l)-\theta C l_{L}^{\frac{1}{2}-\varepsilon} \leq W_{l}, l \in[1, L-1], W_{L} \in H_{y, \delta}\right) \\
& =E_{x}^{W}\left(\prod_{l=1}^{L-2} h_{l}\left(W_{l}, W_{l+1}\right) ; W_{L} \in H_{y, \delta}\right) \\
& \geq E_{x}^{W}\left(\prod_{l=1}^{L-2} h_{l}\left(W_{l}, W_{l+1}\right) ; A_{L} ; W_{L} \in H_{y, \delta}\right) \\
& \geq \prod_{l=1}^{L-2} h_{l}\left(f_{a, b}(l)-C l_{L}^{\frac{1}{2}-\varepsilon}, f_{a, b}(l+1)-C(l+1)_{L}^{\frac{1}{2}-\varepsilon}\right) P_{x}^{W}\left(A_{L} ; W_{L} \in H_{y, \delta}\right) .
\end{aligned}
$$

But using 2.1 with the $L$ there equal to 1 , we have

$$
\begin{aligned}
& \sum_{l=1}^{L-2}\left(1-h_{l}\left(f_{a, b}(l)-C l_{L}^{\frac{1}{2}-\varepsilon}, f_{a, b}(l+1)-C(l+1)_{L}^{\frac{1}{2}-\varepsilon}\right)\right) \\
& \leq \sum_{l=1}^{L-2} \exp \left(-c(\theta-1)^{2} C^{2} l_{L}^{\frac{1}{2}-\varepsilon}\right) \leq \frac{1}{2},
\end{aligned}
$$

if we let $\theta$ be large enough depending on $C$, but independently of $L$. Thus 2.5) follows from (2.3), Remark 2.2 and (2.6).

Remark 2.4. As in Remark 2.2, the condition $|x-y| \leq \eta L$ can be dropped, at the cost of replacing the right hand side of (2.5) by (2.4).

We now derive the corresponding estimates for the 0-dimensional Bessel process by applying a change of measure. To formally introduce the Bessel process recall that the squared Bessel process $Y_{t}^{2}$ is a Markov process, $\mathrm{BESQ}^{0}$, with semigroup, for $x>0$,

$$
V_{t}(x, y)=e^{-x / 2} \delta_{0}(d y)+1_{(0, \infty)}(y) \frac{1}{2 t}\left(\frac{x}{y}\right)^{1 / 2} I_{1}(\sqrt{x y} / t) e^{-(x+y) / 2 t} d y,
$$

see [18, Chapter IX, Corollary (1.4)], where

$$
I_{1}(z)=\sum_{k=0}^{\infty} \frac{(z / 2)^{2 k+1}}{k !(k+1) !},
$$

see [16, 8.447.2], is the first Bessel function. Let $P_{y}^{Y}$ denote the law of the $Y_{t}$ starting at $Y_{0}=z$. From $(2.7)$ the equality 1.12 easily follows. 
Lemma 2.5. For all fixed $\delta>0, \varepsilon \in\left(0, \frac{1}{2}\right)$ and $\eta>1$, there exists $c=$ $c(\delta, \eta, \varepsilon, C)$ so that for $1 \leq x, y, 0 \leq a \leq x, 0 \leq b \leq y$, and $|x-y| \leq \eta L$,

$$
\begin{aligned}
& P_{x}^{Y}\left(f_{a, b}(l ; L)-C l_{L}^{\frac{1}{2}-\varepsilon} \leq Y_{l}, l=1, \ldots, L-1, Y_{L} \in H_{y, \delta}\right) \\
& \leq c \frac{(1+x-a)(1+y-b)}{L} \sqrt{\frac{x}{y L}} e^{-\frac{(x-y)^{2}}{2 L}} .
\end{aligned}
$$

If in addition we assume that $(x-a)(y-b) \leq \eta L$, and $\max (a b,|a-b|) \geq L / \eta$, then for any $\tilde{C} \geq C+\delta>0$ there exists $c^{\prime}=c^{\prime}(\delta, \eta, \varepsilon, C, \tilde{C})$ so that for

$$
\begin{aligned}
& P_{x}^{Y}\left(f_{a, b}(l ; L)+C l_{L}^{\frac{1}{2}-\varepsilon} \leq Y_{l} \leq f_{x, y}(l ; L)+\tilde{C} l_{L}^{\frac{1}{2}+\varepsilon},\right. \\
& \left.\quad l=1, \ldots, L-1, Y_{L} \in H_{y, \delta}\right) \\
& \geq c^{\prime \frac{(1+x-a)(1+y-b)}{L}} \sqrt{\frac{x}{y L}} e^{-\frac{(x-y)^{2}}{2 L}} .
\end{aligned}
$$

Remark 2.6. a) As in Remark 2.2, the condition $|x-y| \leq \eta L$ can be dropped in (2.9), at the cost of replacing the right hand side by

$$
\frac{(1+x-a)(1+y-b)}{L} \sqrt{\frac{x}{y L}} \sup _{z \in H_{y, \delta}} e^{-\frac{(x-z)^{2}}{2 L}} .
$$

b) This is where we need the condition $\max (a b,|a-b|) \geq L / \eta$ from Theorem 1.1 b). It guarantees that

$$
\int_{1}^{L-1} \frac{1}{f_{a, b}^{2}(s ; L)} d s=\frac{L-2}{a b+\frac{(a-b)^{2}}{L}\left(1-\frac{1}{L}\right)} \leq c(\eta)
$$

independent of $L \geq 3$. The bound (2.11) is used below to bound the RadonNikodym derivative of a 0-Bessel process with respect to Brownian motion.

Proof of Lemma 2.5. Recall that $Y_{t}$ solves the SDE $d Y_{t}=d W_{t}-\frac{1}{2 Y_{t}} d t$ for a Brownian motion $W_{t}$, until $\tau_{0}$, the time it hits 0 (not to be confused with the hitting time $\tau_{x}$ of a vertex $x$ of the binary tree, a notation used elsewhere in this paper). By Girsanov's theorem (applied until $Y_{t}$ hits $\epsilon$ ) and monotone convergence it follows that for any $F \in \mathcal{F}_{t}$,

$$
E_{x}^{Y}\left(1_{\left\{\tau_{0}>t\right\}} F\right)=E_{x}^{W}\left(1_{\left\{\tau_{0}>t\right\}} F \sqrt{\frac{x}{W_{t}}} \exp \left(-\frac{3}{8} \int_{0}^{t} \frac{1}{W_{s}^{2}} d s\right)\right) .
$$


We also note that 0 is an absorbing boundary for the 0-dimensional Bessel process, so that $Y_{L}>0$ implies that $\tau_{0}>L$. Therefore, since $y \geq 1$,

$$
\begin{aligned}
\mathcal{I}_{1} & :=P_{x}^{Y}\left(f_{a, b}(l ; L)-C l_{L}^{\frac{1}{2}-\varepsilon} \leq Y_{l}, l=1, \ldots, L-1, Y_{L} \in H_{y, \delta}\right) \\
& =P_{x}^{Y}\left(f_{a, b}(l ; L)-C l_{L}^{\frac{1}{2}-\varepsilon} \leq Y_{l}, l=1, \ldots, L-1, Y_{L} \in H_{y, \delta}, \tau_{0}>L\right) \\
& \leq \sqrt{\frac{x}{y}} P_{x}^{W}\left(f_{a, b}(l ; L)-C l_{L}^{\frac{1}{2}-\varepsilon} \leq W_{l}, l=1, \ldots, L-1, Y_{L} \in H_{y, \delta}\right),
\end{aligned}
$$

by 2.12 . Then using 2.5 ,

$$
\mathcal{I}_{1} \leq c \sqrt{\frac{x}{y} \frac{(1+x-a)(1+y-b)}{L}} \frac{1}{\sqrt{L}} e^{-\frac{(x-y)^{2}}{2 L}} .
$$

This completes the proof of 2.9 .

We turn to the proof of the lower bound 2.10). Letting

$$
\widetilde{A}=\left\{f_{a, b}(l ; L)+C l_{L}^{\frac{1}{2}-\varepsilon} \leq W_{l} \leq f_{x, y}(l ; L)+\tilde{C} l_{L}^{\frac{1}{2}+\varepsilon}, l \in[1, L-1]\right\}
$$

we have, by 2.12 ,

$$
\begin{aligned}
& P_{x}^{Y}\left(\widetilde{A}, Y_{L} \in H_{y, \delta}\right) \geq \sqrt{\frac{x}{y+\delta}} E_{x}^{W}\left(\exp \left(-\frac{3}{8} \int_{0}^{L} \frac{1}{W_{t}^{2}} d t\right) ; \widetilde{A} ; W_{L} \in H_{y, \delta}\right) \\
& \geq c \sqrt{\frac{x}{y}} E_{x}^{W}\left(\exp \left(-\frac{3}{8} \int_{0}^{1} \frac{1}{W_{t}^{2}} d t\right) \cdot \exp \left(-\frac{3}{8} \int_{L-1}^{L} \frac{1}{W_{t}^{2}} d t\right) ; \widetilde{A} ; W_{L} \in H_{y, \delta}\right)
\end{aligned}
$$

where the last inequality follows because on the event $W_{l} \geq f_{a, b}(l ; L)+$ $C l_{L}^{\frac{1}{2}-\varepsilon}, l \in[1, L-1]$, we have $\int_{1}^{L-1} \frac{1}{W_{t}^{2}} d t \leq c(\eta)$ by 2.11 .

Furthermore for any $u, v \geq 1 / \eta$ we have

$$
\begin{aligned}
& J_{u, v}:=E_{u}^{W}\left(\exp \left(-\frac{1}{2} \int_{0}^{1} \frac{1}{W_{t}^{2}} d t\right) \mid W_{1}=v\right) \\
& =E_{0}^{W}\left(\exp \left(-\frac{1}{2} \int_{0}^{1} \frac{1}{\left(W_{t}+u+t(v-u)\right)^{2}} d t\right) \mid W_{1}=0\right) \geq c>0,
\end{aligned}
$$

where the constant depends only on $\eta$, since the standard Brownian bridge has positive probability of staying between $1 / 2 \eta$ and $-1 / 2 \eta$. We apply this to (2.14), where, after the Markov property we need to lower bound $J_{x, W_{1}}$ and $J_{W_{L-1}, v}$ for $v \in[y, y+\delta]$. Since $x, y \geq 1$ and $\max (a b,|a-b|) \geq L / \eta$ implies 
that $f_{a, b}(1 ; L), f_{a, b}(L-1 ; L) \geq 1 / \eta$, we therefore have in fact

$$
\begin{aligned}
& P_{x}^{Y}\left(f_{a, b}(l ; L)+C l_{L}^{\frac{1}{2}-\varepsilon} \leq Y_{l} \leq f_{x, y}(l ; L)+\tilde{C} l_{L}^{\frac{1}{2}+\varepsilon}, l \in[1, L-1], Y_{L} \in H_{y, \delta}\right) \\
& \quad \geq c \sqrt{\frac{x}{y}} P_{x}^{W}\left(\widetilde{A}, ; W_{L} \in H_{y, \delta}\right) \geq c \sqrt{\frac{x}{y} \frac{(1+x-a)(1+y-b)}{L}} \frac{1}{\sqrt{L}} e^{-\frac{(x-y)^{2}}{2 L}}
\end{aligned}
$$

using (2.3). This yields (2.9).

\section{$3 \quad$ Local times and traversal counts}

Let $X_{t}, t \geq 0$, be the continuous time random walk on the weighted graph $\{0,1,2, \ldots\}$ with unit weights on each edge. That is, the continuous time random walk on $\{0,1,2, \ldots\}$ with exponential holding times of mean 1 at 0 and mean $1 / 2$ at all other points, and whose jump chain is simple random walk reflected at the origin. Let $L_{t}^{x}$ denote the local time at $x$ and $\tau(s)=$ $\inf \left\{t>0 \mid L_{t}^{0}=s\right\}$, the inverse local time at 0 . Fix $u>0$ once and for all. We use $\mathbb{P}_{u}$ to denote the probability for the process $\bar{X}_{t}=X_{t \wedge \tau(u)}, t \geq 0$. Let $\mathcal{L}_{l}=L_{\tau(u)}^{l}$, the total local time of $\bar{X}$ at $l$, and let $T_{l}, l \geq 0$, be the discrete traversal count $l \rightarrow l+1$. Under $\mathbb{P}_{u}$ the $T_{l}, l \geq 0$, and $\mathcal{L}_{l}, l \geq 0$, together have a Markovian structure, as stated by the following lemma.

Lemma 3.1 (Markovian structure).

a) The sequence $\left(Z_{n}\right)_{n \geq 0}$ defined by

$$
\left(Z_{0}, Z_{1}, Z_{2}, \ldots\right)=\left(\mathcal{L}_{0}, T_{0}, \mathcal{L}_{1}, T_{1}, \mathcal{L}_{2}, T_{2}, \ldots\right)
$$

is a time inhomogeneous Markov chain under $\mathbb{P}_{u}$. When $n=2 k$ is even the law of $Z_{n+1}=T_{k}$ conditioned on $Z_{n}=\mathcal{L}_{k}=v$ is Poisson with parameter $v$, and when $n=2 k+1$ is odd the law of $Z_{n+1}=\mathcal{L}_{k+1}$ conditioned on $Z_{n}=T_{k}=m$ is 0 if $m=0$ and is the gamma distribution with shape $m$ and scale parameter 1 if $m \geq 1$.

b) Under $\mathbb{P}_{u}\left(\cdot \mid T_{1}=m_{1}, \ldots, T_{L}=m_{L}\right)$ the $\mathcal{L}_{1}, \ldots, \mathcal{L}_{L+1}$ are independent, $\mathcal{L}_{l}$ depends only on $T_{l-1}$ and $T_{l}$, and the $\mathbb{P}_{u}\left(\cdot \mid T_{l-1}=m_{l-1}, T_{l}=m_{l}\right)$-law of $\mathcal{L}_{l}$ is the gamma distribution with shape parameter $m_{l-1}+m_{l}$ and scale parameter $\frac{1}{2}$.

c) Under $\mathbb{P}_{u}\left(\cdot \mid \mathcal{L}_{1}=y_{1}, \ldots, \mathcal{L}_{L+1}=y_{L+1}\right)$ the $T_{1}, \ldots, T_{L}$ are independent, $T_{l}$ depends only on $\mathcal{L}_{l}$ and $\mathcal{L}_{l+1}$, and with $u_{l}, u_{l+1}>0$,

$$
\mathbb{P}_{u}\left(T_{l}=m \mid \mathcal{L}_{l}=u_{l}, \mathcal{L}_{l+1}=u_{l+1}\right)=\frac{\left(u_{l} u_{l+1}\right)^{m} /((m-1) ! m !)}{\sqrt{u_{l} u_{l+1}} I_{1}\left(2 \sqrt{u_{l} u_{l+1}}\right)} .
$$


d) $\left(T_{l}\right)_{l>0}$ under $\mathbb{P}_{u}$ is a Markov chain with the same transition kernel as that of $\left(T_{l}\right)_{l \geq 0}$ under $P$..

e) $\left(\mathcal{L}_{l}\right)_{l \geq 0}$ under $\mathbb{P}_{u}$ is $\frac{1}{2} B E S Q^{0}(2 u)$ at integer times. That is, $\left(\mathcal{L}_{l}\right)_{l \geq 0}$ under $\mathbb{P}_{u}$ is a Markov chain with the same transition kernel as $\left(\frac{1}{2} Y_{l}^{2}\right)_{l \geq 0}$ under $P^{Y}$.

Remark 3.2. The distribution in (3.1) is a generalization to $\nu=-1$ of the Bessel distribution Bessel $(\nu, z)$ defined in 17 for $\nu>-1$. This distribution appears in [15]. See also [19].

Proof of Lemma 3.1. Construct a collection of independent standard Poisson processes, one for each directed edge. The random walk is constructed by placing a local time clock at each vertex which only advances when the walker is at that vertex. When the walker is at a given vertex its local time clock advances until one of the two independent Poisson processes associated with edges originating at the vertex register an arrival, and then the walker traverses the corresponding edge. Note that the minimum of two independent exponentials of mean 1 is an exponential of mean $1 / 2$. From this construction it is clear that given $u$ the count $T_{0}$ is the number of arrivals up to time $u$ of the point process associated to the edge $0 \rightarrow 1$, and therefore is Poisson with parameter $u$. Given $T_{0}=m$, then $\mathcal{L}_{1}$, the amount of local time spent at vertex 1 until the walker has returned to 0 a total of $m$ times, is the time until the $m$-th arrival of the Poisson process associated to the edge $1 \rightarrow 0$, and therefore gamma distributed with shape $m$ and scale parameter 1 . Iterating this a) follows and d) is immediate.

b) It follows from the fact that $Z_{n}$ is a Markov chain that under $\mathbb{P}_{u}\left(\cdot \mid T_{1}=m_{1}, \ldots, T_{L}=m_{L}\right)$ the $\mathcal{L}_{1}, \ldots, \mathcal{L}_{L+1}$ are independent and that the conditional law of $\mathcal{L}_{l}$ depends only on $T_{l-1}$ and $T_{l}$. The characterization of the conditional law follows immediately since each local time $\mathcal{L}_{l}$ is the sum of independent mean $1 / 2$ exponentials, one for every visit to $l$, and $m_{l-1}+m_{l}$ is the number of such visits.

c) Similarly to above the conditional independence and dependence of the conditional law of $T_{l}$ only on $\mathcal{L}_{l}$ and $\mathcal{L}_{l+1}$ follows from the Markovian structure. From the joint law, with $u_{l}>0$ and $m_{l} \geq 1$,

$$
\begin{aligned}
\mathbb{P}_{u}\left(T_{l}=m_{l}, \mathcal{L}_{l+1}=d u_{l+1} \mid \mathcal{L}_{l}=u_{l}\right) & =e^{-u_{l}} \frac{u_{l}^{m_{l}}}{m_{l} !} \times \frac{u_{l+1}^{m_{l}-1}}{\left(m_{l}-1\right) !} e^{-u_{l+1}}, \\
& =\frac{1}{u_{l+1}} \times \frac{\left(u_{l} u_{l+1}\right)^{m_{l}}}{m_{l} !\left(m_{l}-1\right) !} e^{-\left(u_{l}+u_{l+1}\right)}
\end{aligned}
$$


while

$$
\mathbb{P}_{u}\left(T_{l}=0, \mathcal{L}_{l+1}=d u_{l+1} \mid \mathcal{L}_{l}=u_{l}\right)=e^{-u_{l}} \delta_{0}\left(d u_{l+1}\right) .
$$

Hence, using (2.8), we obtain

$$
\begin{aligned}
& \mathbb{P}_{u}\left(\mathcal{L}_{l+1}=d u_{l+1} \mid \mathcal{L}_{l}=u_{l}\right) \\
& =e^{-u_{l}} \delta_{0}\left(d u_{l+1}\right)+\frac{1}{u_{l+1}} \sum_{m \geq 1} \frac{\left(u_{l} u_{l+1}\right)^{m}}{(m-1) ! m !} e^{-\left(u_{l}+u_{l+1}\right)} \\
& =e^{-u_{l}} \delta_{0}\left(d u_{l+1}\right)+\left(\frac{u_{l}}{u_{l+1}}\right)^{1 / 2} I_{1}\left(2 \sqrt{u_{l} u_{l+1}}\right) e^{-\left(u_{l}+u_{l+1}\right)},
\end{aligned}
$$

and one concludes that for $m \geq 1$, which is equivalent to $u_{l+1}>0$,

$$
\mathbb{P}_{u}\left(T_{l}=m \mid \mathcal{L}_{l}=u_{l}, \mathcal{L}_{l+1}=u_{l+1}\right)=\frac{\left(u_{l} u_{l+1}\right)^{m} /((m-1) ! m !)}{\sqrt{u_{l} u_{l+1}} I_{1}\left(2 \sqrt{u_{l} u_{l+1}}\right)} .
$$

e) This follows by comparing (3.3) with 2.7).

We will use $\mathbb{Q}$ to denote the law of the time inhomogeneous Markov chain $\left(Z_{n}\right)_{n \geq 0}$ under $\mathbb{P}_{u}$. For $F \in \sigma\left(Z_{n}, Z_{n+1}, \ldots\right)$ let

$$
\mathbb{Q}_{n}^{x}=\mathbb{Q}\left(F \mid Z_{n}=x\right) .
$$

Thus $\mathbb{Q}=\mathbb{Q}_{0}^{u}$.

For future reference we restate part e) of Lemma 3.1 as

$$
\sqrt{2 Z_{2 l}}, \quad l=0,1, \ldots \text { under } \mathbb{Q} \text { is a }\left.\operatorname{Bes}^{0}(\sqrt{2 u})\right|_{\mathbb{N}} .
$$

In the following we often replace the $Z$ 's by the equivalent $\mathcal{L}$ 's and $T$ 's.

The following lemma gives some estimates for one step transitions of the Markov chain.

Lemma 3.3 (One step estimates).

a) For all $x>0$ such that $x^{2} / 2$ is an integer and all $l \geq 1$,

$$
\begin{aligned}
& \mathbb{Q}_{2 l-1}^{x^{2} / 2}\left(\left|\sqrt{2 Z_{2 l}}-x\right| \geq z\right) \\
& =\mathbb{Q}\left(\left|\sqrt{2 \mathcal{L}_{l}}-x\right| \geq z \mid \sqrt{2 T_{l-1}}=x\right) \leq e^{-c z^{2}} .
\end{aligned}
$$


b) For all $-\sqrt{2} \leq a<b$,

$$
\inf _{\frac{x^{2}}{2} \in\{1,2, \ldots\}} \mathbb{Q}\left(\sqrt{2 \mathcal{L}_{l}}-x \in[a, b] \mid \sqrt{2 T_{l-1}}=x\right) \geq c>0,
$$

with a constant $c$ depending only on $a, b$.

c) For all $x>0$, and all $l \geq 1$,

$$
\begin{aligned}
& \mathbb{Q}_{2 l}^{x^{2} / 2}\left(\left|\sqrt{2 Z_{2 l+1}}-x\right| \geq z\right) \\
& =\mathbb{Q}\left(\left|\sqrt{2 T_{l}}-x\right| \geq z \mid \sqrt{2 \mathcal{L}_{l}}=x\right) \leq e^{-c z^{2}} .
\end{aligned}
$$

d) For all $-1 \leq a<b$,

$$
\inf _{x \geq 1: x+[a, b] \cap \sqrt{2 \mathbb{N}} \neq \emptyset} \mathbb{Q}\left(\sqrt{2 T_{l}}-x \in[a, b] \mid \sqrt{2 \mathcal{L}_{l}}=x\right) \geq c>0,
$$

with a constant $c$ depending only on $a, b$.

Proof of Lemma 3.3. Standard large deviation bounds for the gamma and Poisson distributions give $(3.6)$ and $(3.8)$. Indeed, to see $(3.8)$, recall that by Lemma $3.1 \mathrm{a}$ ), the conditional law of $T_{l}$, when conditioned on $\mathcal{L}_{l-1}=x^{2} / 2$, is Poisson of parameter $x^{2} / 2$. Since the Legendre transform of the logarithmic moment generating function of the Poisson distribution of parameter $\lambda$ is

$$
I_{P, \lambda}(x)=\lambda-x+x \log (x / \lambda),
$$

we obtain from Chebyshev's inequality that, for $z \geq 0$,

$$
\begin{aligned}
\log & \mathbb{Q}\left(\sqrt{2 T_{l}}-x \geq z \mid \sqrt{2 \mathcal{L}_{l}}=x\right) \leq-I_{P, x^{2} / 2}\left(\frac{(z+x)^{2}}{2}\right) \\
& =-\left[\frac{x^{2}}{2}+\frac{(x+z)^{2}}{2}\left(2 \log \left(1+\frac{z}{x}\right)-1\right)\right]
\end{aligned}
$$

and

$$
\begin{aligned}
\log & \mathbb{Q}\left(\sqrt{2 T_{l}}-x \leq-z \mid \sqrt{2 \mathcal{L}_{l}}=x\right) \leq-I_{P, x^{2} / 2}\left(\frac{(x-z)^{2}}{2}\right) \\
= & -\left[\frac{x^{2}}{2}+\frac{(x-z)^{2}}{2}\left(2 \log \left(1-\frac{z}{x}\right)-1\right)\right] .
\end{aligned}
$$

Separating according to whether $z / x>1$ (only relevant for (3.11)) or $z / x \leq 1$, and performing some algebra yields (3.8). The argument for (3.6) is similar: 
recall, again from Lemma 3.1 a), that the conditional law of $\mathcal{L}_{l}$, when conditioned on $T_{l-1}=x^{2} / 2$, is gamma of shape parameter $x^{2} / 2$ and scale parameter 1. Recall that the Legendre transform of the logarithmic moment generating function of a Gamma variable of shape parameter $m$ and scale parameter 1 equals

$$
I_{\Gamma, \lambda}(x)=x-m+m \log (m / x) .
$$

Replacing the expression in (3.10) with (3.13) and then using Chebyshev's inequality to obtain the analogue of (3.11) and (3.12) (with $m=x^{2} / 2$ ), the proof then proceeds similarly to the Poisson case.

To see (3.7), note that by the central limit theorem for a sum of independent exponentials,

$$
\lim _{x \rightarrow \infty, \frac{x^{2}}{2} \in\{1,2, \ldots\}} \mathbb{Q}_{1}^{x^{2} / 2}\left(\mathcal{L}_{1} \in \frac{1}{2}\left[(x+a)^{2},(x+b)^{2}\right]\right)=\int_{a}^{b} \frac{1}{\sqrt{4 \pi}} e^{-\frac{u^{2}}{4}} d u>0 .
$$

Thus for a large enough $\tilde{c}>0$ we have for $x^{2} / 2 \geq \tilde{c}$ that

$$
\mathbb{Q}_{1}^{x^{2} / 2}\left(\sqrt{2 \mathcal{L}_{1}}-x \in[a, b]\right) \geq c>0 .
$$

Since the density $f(z)$ of a gamma random variable with shape parameter $x^{2} / 2$ and scale parameter 1 satisfies $f(z) \geq c>0$ for all $0 \leq z \leq \frac{1}{2}(\tilde{c}+b)^{2}$ if $1 \leq x^{2} / 2 \leq \tilde{c}$, it follows that the same holds for $1 \leq x^{2} / 2 \leq \tilde{c}$. This proves (3.7). Similarily (3.9) follows from the central limit theorem for the Poisson distribution, and since by our assumptions on $[a, b]$ imply that the interval always contains an element from $\sqrt{2 \mathbb{N}}$.

The following lemma gives some estimates for conditioning the Markov chain at two times, before and after.

Lemma 3.4 (Local time and transversal bounds).

a) For all $m_{l-1}, m_{l}$ and $0 \leq z \leq \sqrt{m_{l-1}+m_{l}}$,

$$
\mathbb{Q}_{1}^{m_{0}}\left(\sqrt{2 \mathcal{L}_{l}} \geq \sqrt{m_{l-1}+m_{l}}-z \mid T_{l-1}=m_{l-1}, T_{l}=m_{l}\right) \geq 1-\exp \left(-z^{2}\right) .
$$

b) For all $-\sqrt{2} \leq a<b$,

$$
\inf _{\substack{t_{l-1}>0 \\ t_{l} \geq 0}} \mathbb{Q}_{1}^{m_{0}}\left(\sqrt{2 \mathcal{L}_{l}}-\sqrt{m_{l-1}+m_{l}} \in[a, b] \mid T_{l-1}=m_{l-1}, T_{l}=m_{l}\right) \geq c,
$$

for all $l$, and a constant $c>0$ depending only on $a, b$. 
c) For all $u_{l}, u_{l+1}$ and $z \geq 0$

$$
\mathbb{Q}_{1}^{m_{0}}\left(\left|\sqrt{2 T_{l}}-\left(4 u_{l} u_{l+1}\right)^{1 / 4}\right| \geq z \mid \mathcal{L}_{l}=u_{l}, \mathcal{L}_{l+1}=u_{l+1}\right) \leq c \exp \left(-c z^{2}\right) .
$$

d) For all $a<b$, with

$$
D_{a, b}=\left\{u_{l}>0, u_{l+1} \geq 0 \mid\left(u_{l} u_{l+1}\right)^{1 / 4}+[a, b] \cap \sqrt{2 \mathbb{N}} \neq \emptyset\right\},
$$

we have

$$
\inf _{u_{l}, u_{l+1} \in D_{a, b}} \mathbb{Q}_{1}^{m_{0}}\left(\sqrt{2 T_{l}}-\left(4 u_{l} u_{l+1}\right)^{1 / 4} \in[a, b] \mid \mathcal{L}_{l}=u_{l}, \mathcal{L}_{l+1}=u_{l+1}\right) \geq c,
$$

for some $c>0$ depending only on $a, b$.

Proof. Similarly to (3.7), the claim (3.15) follow from the central limit theorem for a sum of exponential random variables, since under the conditioning $\mathcal{L}_{l}$ have the gamma distribution with an integer shape parameter (see Lemma 3.1 b). A large deviation bound for the gamma distribution that can be proved using the exponential Chebyshev inequality gives

$$
\mathbb{Q}_{1}^{m_{0}}\left(\mathcal{L}_{l} \leq \frac{m_{l-1}+m_{l}}{2}-a \mid T_{1}=m_{1}, \ldots, T_{L}=m_{L}\right) \leq \exp \left(-2 \frac{a^{2}}{m_{l-1}+m_{l}}\right)
$$

for $0 \leq a \leq \frac{m_{l-1}+m_{l}}{2}$. By taking $a=z \sqrt{\frac{m_{l-1}+m_{l}}{2}}$ this implies 3.14 .

A basic large deviation estimate for the Bessel distribution (see [6, Lemma 7.12] and its proof)

$$
\begin{aligned}
& \mathbb{Q}_{1}^{m_{0}}\left(\left|T_{l}-\sqrt{u_{l} u_{l+1}}\right| \geq a \mid \mathcal{L}_{l}=u_{l}, \mathcal{L}_{l+1}=u_{l+1}\right) \\
& \quad \leq c \exp \left(-c \frac{a^{2}}{\sqrt{u_{l} u_{l+1}}}+c \frac{a}{\sqrt{u_{l} u_{l+1}}}\right) \text { for all } a \geq 0 .
\end{aligned}
$$

By taking $a=z \sqrt{u_{l} u_{l+1}}$ this implies $(3.16)$.

For 3.17), set $z=\sqrt{u_{l} u_{l+1}}$ and $m=\frac{(\sqrt{2 z}+v)^{2}}{2}$ in 3.1 and use the estimate $I_{1}(z) \sim \frac{e^{\omega}}{\sqrt{2 \pi z}}$ for $z \rightarrow \infty$, see [16, 8.451.5], and Stirling's formula to see that for $v \in[a, b]$ and $z \geq z_{0}$ large enough

$$
\mathbb{Q}_{1}^{m_{0}}\left(\sqrt{2 T_{l}}=\sqrt{2 z}+v \mid \mathcal{L}_{l}=u_{l}, \mathcal{L}_{l+1}=u_{l+1}\right) \geq c \frac{e^{-3 v^{2}}}{\sqrt{z}} .
$$


There are at least $c \sqrt{z}$ integers of the form $m=\frac{(\sqrt{2 z}+v)^{2}}{2}$ for $v \in[a, b]$, so one obtains (3.17) for $z \geq z_{0}$. For smaller $z \leq z_{0}$ one simply uses the fact that the right-hand side of (3.1) is bounded away from zero for the finite number of $m=\frac{(\sqrt{2 z}+v)^{2}}{2}$ with $v \in[a, b]$ and $u_{l}, u_{l+1} \in D_{a, b}$.

\section{Galton-Watson process proofs}

We now have the necessary tools to prove the precise large deviation estimate Proposition 1.4 and the barrier estimate Theorem 1.1 for the process $T_{l}$. We start with the former.

Proof of Proposition 1.4. Since $T_{L}=0$ is the event that none of $x^{2} / 2$ independent excursions from 1 to 0 of a simple random walk on $\{0,1,2, \ldots\}$ hit $L+1$, and this has probability $1-1 /(L+1)$, the estimate (1.11) follows using the assumption $x \leq \eta L$.

Turning to $(1.9)$, we use that

$$
\mathbb{Q}_{1}^{x^{2} / 2}\left(\sqrt{2 T_{L}} \in H_{y, \delta}\right) \leq \frac{\mathbb{Q}_{1}^{x^{2} / 2}\left(\sqrt{2 \mathcal{L}_{L+1}} \in H_{y, 2 \delta}\right)}{\mathbb{Q}_{1}^{x^{2} / 2}\left(\sqrt{2 \mathcal{L}_{L+1}} \in H_{y, 2 \delta} \mid \sqrt{2 T_{L}} \in H_{y, \delta}\right)} .
$$

By (3.7) we have that

$$
\mathbb{Q}_{1}^{x^{2} / 2}\left(\sqrt{2 \mathcal{L}_{L+1}} \in H_{y, 2 \delta} \mid \sqrt{2 T_{L}} \in H_{y, \delta}\right) \geq c>0,
$$

for a constant $c$ depending only on $\delta$. Also

$$
\mathbb{Q}_{1}^{x^{2} / 2}\left(\sqrt{2 \mathcal{L}_{L}} \in H_{y, \delta / 2}\right) \leq \frac{\mathbb{Q}_{1}^{x^{2} / 2}\left(\sqrt{2 T_{L}} \in H_{y, \delta}\right)}{\mathbb{Q}_{1}^{x^{2} / 2}\left(\sqrt{2 T_{L}} \in H_{y, \delta} \mid \sqrt{2 \mathcal{L}_{L}} \in H_{y, \delta / 2}\right)},
$$

and by 3.9

$$
\mathbb{Q}_{1}^{x^{2} / 2}\left(\sqrt{2 T_{L}} \in H_{y, \delta} \mid \sqrt{2 \mathcal{L}_{L}} \in H_{y, \delta / 2}\right) \geq c>0,
$$

for a constant $c$ depending only on $\delta$. Thus we have shown that

$$
\begin{aligned}
c \mathbb{Q}_{1}^{x^{2} / 2}\left(\sqrt{2 \mathcal{L}_{L}} \in H_{y, \delta / 2}\right) & \leq \mathbb{Q}_{1}^{x^{2} / 2}\left(\sqrt{2 T_{L}} \in H_{y, \delta}\right) \\
& \leq \frac{1}{c} \mathbb{Q}_{1}^{x^{2} / 2}\left(\sqrt{2 \mathcal{L}_{L+1}} \in H_{y, 2 \delta}\right) .
\end{aligned}
$$


From Lemma $3.1 \mathrm{c}$ ) and 1.12 one obtains

$$
\begin{aligned}
\mathbb{Q}_{1}^{x^{2} / 2}\left(\sqrt{2 \mathcal{L}_{L+1}} \in H_{y, 2 \delta}\right) & =\mathbb{Q}_{1}^{x^{2} / 2}\left(\mathbb{Q}_{2}^{\mathcal{L}_{1}}\left(\sqrt{2 \mathcal{L}_{L}} \in H_{y, 2 \delta}\right)\right) \\
& =\mathbb{Q}_{1}^{x^{2} / 2}\left(P_{\sqrt{2 \mathcal{L}_{1}}}^{Y}\left(Y_{L} \in H_{y, 2 \delta}\right)\right) \\
& =\mathbb{Q}_{1}^{x^{2} / 2}\left(\int_{y}^{y+2 \delta} \frac{\sqrt{2 \mathcal{L}_{1}}}{L} e^{-\frac{2 \mathcal{L}_{1}+z^{2}}{2 L}} I_{1}\left(\frac{\sqrt{2 \mathcal{L}_{1}} z}{L}\right) d z\right) .
\end{aligned}
$$

The function $I_{1}(z)$, see $(2.8)$, is continuous, and clearly non-zero for $z>0$, $I_{1}(z) \sim z / 2$ for $z \rightarrow 0$ and $I_{1}(z) \sim \frac{e^{z}}{\sqrt{2 \pi z}}$ for $z \rightarrow \infty$, see [16, 8.451.5]. In particular, $I_{1}(z) \leq C \frac{e^{z}}{\sqrt{z}}$. Using our assumptions on $x, y$ and 3.6 , one sees that

$$
\mathbb{Q}_{1}^{x^{2} / 2}\left(\sqrt{2 \mathcal{L}_{L+1}} \in H_{y, 2 \delta}\right) \leq c \sqrt{\frac{x / y}{L}} e^{-\frac{x^{2}+y^{2}}{2 L}} e^{\frac{x y}{L}} .
$$

Similarly, since $I_{1}(z) \geq C^{\prime} \frac{e^{z}}{\sqrt{z}}$ for $z \geq \delta>0$, it follows from $\sqrt{3.6}$ that if $L / \eta \leq x y$

$$
\mathbb{Q}_{1}^{x^{2} / 2}\left(\sqrt{2 \mathcal{L}_{L}} \in H_{y, \delta / 2}\right) \geq c^{\prime} \sqrt{\frac{x / y}{L}} e^{-\frac{x^{2}+y^{2}}{2 L}} e^{\frac{x y}{L}} .
$$

Together with (4.1) this proves (1.9).

Proof of Theorem 1.1. We begin with the proof of the upper bound (1.1). Fix $C>0$ and let

$$
A=\left\{f_{a, b}(l ; L)-C l_{L}^{\frac{1}{2}-\varepsilon} \leq \sqrt{2 T_{l}}, l=1, \ldots, L-1, \sqrt{2 T_{L}} \in H_{y, \delta}\right\} .
$$

We will first prove that

$$
\mathbb{Q}_{1}^{x^{2} / 2}(A) \leq c \frac{(1+x-a)(1+y-b)}{L^{3 / 2}} \sqrt{\frac{x}{y}} e^{-\frac{(x-y)^{2}}{2 L}} \text { for all } y \geq \sqrt{2} .
$$

Consider the event

$$
\begin{gathered}
B=\left\{\sqrt{2 \mathcal{L}_{1}} \geq a^{\prime}+\frac{x-a}{2}, \sqrt{2 \mathcal{L}_{l}} \geq f_{a^{\prime} b}(l-1 ; L-1)-\bar{C}(l-1)_{L-1}^{\frac{1}{2}-\varepsilon},\right. \\
\text { for } \left.l=2, \ldots, L-1 ; \sqrt{2 \mathcal{L}_{L}} \geq \frac{y+b}{2}\right\}
\end{gathered}
$$

for a fixed $\bar{C} \geq 1$ to be specified later, where

$$
a^{\prime}=f_{a, b}(1 ; L) \text {, so that } f_{a^{\prime} b}(l-1 ; L-1)=f_{a, b}(l ; L) .
$$


By Lemma 3.1 b),

$$
\begin{aligned}
& \mathbb{Q}_{1}^{x^{2} / 2}(B \cap A)=\mathbb{Q}_{1}^{x^{2} / 2}\left\{1_{A} \mathbb{Q}_{1}^{x^{2} / 2}\left(\sqrt{2 \mathcal{L}_{1}} \geq a^{\prime}+\frac{x-a}{2} \mid T_{0}, T_{1}\right)\right. \\
& \times\left(\prod_{l=2}^{L-1} \mathbb{Q}_{1}^{x^{2} / 2}\left(\sqrt{2 \mathcal{L}_{l}} \geq f_{a^{\prime} b}(l-1 ; L-1)-\bar{C}(l-1)_{L-1}^{\frac{1}{2}-\varepsilon} \mid T_{l-1}, T_{l}\right)\right) \\
& \left.\times \mathbb{Q}_{1}^{x^{2} / 2}\left(\sqrt{2 \mathcal{L}_{L}} \geq \frac{y+b}{2} \mid T_{L-1}, T_{L}\right)\right\} .
\end{aligned}
$$

On the event $A$ we have

$$
\sqrt{T_{0}+T_{1}} \geq \frac{\sqrt{T_{0}}+\sqrt{T_{1}}}{\sqrt{2}} \geq \frac{x+a^{\prime}-C}{2} \geq a^{\prime}+\frac{x-a}{2}-C-C^{\prime},
$$

for a constant $C^{\prime}$ depending only on $\eta$, where we used that $\left|a^{\prime}-a\right| \leq \eta$ by our assumptions on $x, y$. Thus we have by (3.15) with say $\max \left(C+C^{\prime}, 0\right)$ in place of $a$ and $\max \left(C+C^{\prime}, 0\right)+1$ in place of $b$ that for some $c_{1}=c_{1}(C, \eta)>0$,

$$
1_{A} \mathbb{Q}_{1}^{x^{2} / 2}\left(\sqrt{2 \mathcal{L}_{1}} \geq a^{\prime}+\frac{x-a}{2} \mid T_{0}, T_{1}\right) \geq 1_{A} c_{1} .
$$

Similarly for some $c_{2}>0$,

$$
1_{A} \mathbb{Q}_{1}^{x^{2} / 2}\left(\sqrt{2 \mathcal{L}_{L}} \geq \frac{y+b}{2} \mid T_{L-1}, T_{L}\right) \geq 1_{A} c_{2} .
$$

Note that for any $l^{\prime} \leq L^{\prime}$ with $L / 2 \leq L^{\prime} \leq 2 L$,

$f_{a, b}(l ; L)=f_{a, b}\left(l^{\prime} ; L^{\prime}\right)+(b-a)\left(\frac{l}{L}-\frac{l^{\prime}}{L^{\prime}}\right)=f_{a, b}\left(l^{\prime} ; L^{\prime}\right)+O_{\eta}\left(\left|l^{\prime}-l\right|+\left|L-L^{\prime}\right|\right)$,

and

$$
l_{L}^{\frac{1}{2}-\varepsilon}=\left(l_{L^{\prime}}^{\prime}\right)^{\frac{1}{2}-\varepsilon}+O_{\eta}\left(\left|l-l^{\prime}\right|+\left|L-L^{\prime}\right|\right),
$$

which implies that on the event $A$,

$$
\sqrt{\frac{T_{l-1}+T_{l}}{2}} \geq \min \left(\sqrt{T_{l-1}}, \sqrt{T_{l}}\right) \geq \frac{1}{\sqrt{2}}\left\{f_{a, b}(l ; L)-C l_{L}^{\frac{1}{2}-\varepsilon}-c\right\},
$$

for $2 \leq l \leq L-1$ for a constant $c$ depending only on $\eta$ and $C$. Since $f_{a, b}(l ; L)=$ $f_{a^{\prime}, b}(l-1 ; L-1)$ and $l_{L},(l-1)_{L-1} \geq 1$ for $2 \leq l \leq L-1$ we have if we pick $\bar{C}$ large enough that on $A$,

$$
\sqrt{\frac{T_{l-1}+T_{l}}{2}} \geq \frac{1}{\sqrt{2}}\left\{f_{a^{\prime} b}(l-1 ; L-1)-\frac{\bar{C}}{2}(l-1)_{L-1}^{\frac{1}{2}-\varepsilon}\right\}
$$


for $2 \leq l \leq L-1$. Then by (3.14) we have

$$
\begin{aligned}
& 1_{A} \prod_{l=2}^{L-1} \mathbb{Q}_{1}^{x^{2} / 2}\left(\sqrt{2 \mathcal{L}_{l}} \geq f_{a^{\prime} b}(l-1 ; L-1)-\bar{C}(l-1)_{L-1}^{\frac{1}{2}-\varepsilon} \mid T_{l-1}, T_{l}\right) \\
& \geq 1_{A} \prod_{l=2}^{L-1}\left(1-\exp \left(-\bar{C}^{2}(l-1)_{L-1}^{1-\varepsilon}\right)\right) \geq \frac{1_{A}}{2}
\end{aligned}
$$

where the last inequality follows by making $\bar{C}$ large enough (the choice can be made independent of $L$ ). Combining (4.4), (4.5), (4.6) and (4.9) we obtain $c \mathbb{Q}_{1}^{x^{2} / 2}(A) \leq \mathbb{Q}_{1}^{x^{2} / 2}(A \cap B)$ with $c=\frac{1}{2} c_{1} c_{2}>0$, so that

$$
c \mathbb{Q}_{1}^{x^{2} / 2}(A) \leq \mathbb{Q}_{1}^{x^{2} / 2}(A \cap B) \leq \mathbb{Q}_{1}^{x^{2} / 2}\left(B, \sqrt{2 T_{L}} \in H_{y, \delta}\right) .
$$

Recalling that $T_{k}=Z_{2 k+1}$ and $\mathcal{L}_{k}=Z_{2 k}$, the right-hand side can be written as

$$
\begin{gathered}
J_{1}:=\mathbb{Q}_{1}^{x^{2} / 2}\left(\sqrt{2 Z_{2}} \geq a^{\prime}+\frac{x-a}{2}, \sqrt{2 Z_{2 l}} \geq f_{a^{\prime} b}(l-1 ; L-1)-\bar{C}(l-1)_{L-1}^{\frac{1}{2}-\varepsilon},\right. \\
\left.\quad \text { for } l=2, \ldots, L-1 ; \sqrt{2 Z_{2 L}} \geq \frac{y+b}{2}, \sqrt{2 Z_{2 L+1}} \in H_{y, \delta}\right)
\end{gathered}
$$

Using the Markov property

$$
\begin{gathered}
J_{1}=\mathbb{Q}_{1}^{x^{2} / 2}\left(\sqrt{2 Z_{2}} \geq a^{\prime}+\frac{x-a}{2} ;\right. \\
\mathbb{Q}_{2}^{Z_{2}}\left(\sqrt{2 Z_{2 l}} \geq f_{a^{\prime} b}(l-1 ; L-1)-\bar{C}(l-1)_{L-1}^{\frac{1}{2}-\varepsilon}, l=2, \ldots, L-1 ;\right. \\
\left.\left.\sqrt{2 Z_{2 L}} \geq \frac{y+b}{2} ; \mathbb{Q}_{2 L}^{Z_{2 L}}\left(\sqrt{2 Z_{2 L+1}} \in H_{y, \delta}\right)\right)\right) .
\end{gathered}
$$

By 3.6 , for all $j \geq a^{\prime}+\frac{x-a}{2}$,

$$
\mathbb{Q}_{1}^{x^{2} / 2}\left(\sqrt{2 Z_{2}} \in H_{j}\right) \leq e^{-c(j-x)^{2}},
$$

and by 3.8 , for all $k \geq \frac{y+b}{2}$,

$$
\sup _{v \in H_{k}} \mathbb{Q}_{2 L}^{v^{2} / 2}\left(\sqrt{2 Z_{2 L+1}} \in H_{y, \delta}\right) \leq e^{-c(k-y)^{2}} .
$$


This shows that

$$
\begin{array}{r}
J_{1} \leq \sum_{j \geq a^{\prime}+\frac{x-a}{2}, k \geq \frac{y+b}{2}} \sup _{z \in H_{j}} \mathbb{Q}_{2}^{z^{2} / 2}\left(\sqrt{2 Z_{2 l}} \geq f_{a^{\prime} b}(l-1 ; L-1)-\bar{C}(l-1)_{L-1}^{\frac{1}{2}-\varepsilon},\right. \\
\text { for } \left.l=2, \ldots, L-1 ; \sqrt{2 Z_{2 L}} \in H_{k}\right) e^{-c(j-x)^{2}} e^{-c(k-y)^{2}} .
\end{array}
$$

By 3.5 then

$$
\begin{aligned}
& K_{1}:=\mathbb{Q}_{2}^{z^{2} / 2}\left(\sqrt{2 Z_{2 l}} \geq f_{a^{\prime} b}(l-1 ; L-1)-\bar{C}(l-1)_{L-1}^{\frac{1}{2}-\varepsilon},\right. \\
& \text { for } \left.l=2, \ldots, L-1 ; \sqrt{2 Z_{2 L}} \in H_{k}\right) \\
& =P_{z}^{Y}\left(Y_{l-1} \geq f_{a^{\prime} b}(l-1 ; L-1)-\bar{C}(l-1)_{L-1}^{\frac{1}{2}-\varepsilon}\right. \text {, } \\
& \text { for } \left.l=2, \ldots, L-1 ; Y_{L-1} \in H_{k}\right) \text {, }
\end{aligned}
$$

where $Y_{t}$ is $\operatorname{ans}^{0}(\sqrt{2 u})$ process. Thus using 2.9 we obtain that if $j, k \geq 1$ and $|j-k| \leq 2 \eta L$

$$
\begin{aligned}
& K_{1}=P_{z}^{Y}\left(Y_{l} \geq f_{a^{\prime} b}(l ; L-1)-\bar{C} l_{L-1}^{\frac{1}{2}-\varepsilon},\right. \\
& \text { for } \left.l=1, \ldots, L-2 ; Y_{L-1} \in H_{k}\right) \\
& \leq C \frac{\left(1+z-a^{\prime}\right)(1+k-b)}{(L-1)^{3 / 2}} \sqrt{\frac{z}{k}} e^{-\frac{(z-k)^{2}}{2(L-1)}}
\end{aligned}
$$

Bounding $K_{1}$ by 1 when $|j-k|>2 \eta L$, it then follows that $J_{1}$ is bounded by

$$
\begin{aligned}
& c \sum_{\substack{j \geq a^{\prime}+\frac{x-a}{2} \\
k \geq \frac{y+b}{2},|j-k| \leq 2 \eta L}} \frac{\left(1+j-a^{\prime}\right)(1+k-b)}{(L-1)^{3 / 2}} \sqrt{\frac{j}{k}} e^{-\frac{(j-k)^{2}}{2(L-1)}} e^{-c(j-x)^{2}} e^{-c(k-y)^{2}} \\
& +\sum_{\substack{j \geq a^{\prime}+\frac{x-a}{2} \\
k \geq \frac{y+b}{2},|j-k|>2 \eta L}} e^{-c(j-x)^{2}} e^{-c(k-y)^{2}} .
\end{aligned}
$$

The first sum is bounded by

$$
c \frac{\left(1+\frac{x-a}{2}\right)\left(1+\frac{y-b}{2}\right)}{L^{3 / 2}} \sqrt{\frac{x}{y}} e^{-\frac{(x-y)^{2}}{2 L}} .
$$


As for the second sum, our assumption that $x, y \leq \eta L$ together with $|j-k|>$ $2 \eta L$ implies that either $|x-j| \geq \eta L$ or $|y-k| \geq \eta L$, (or both), in which case the second sum in 4.17) is bounded by $c e^{-c^{\prime} L^{2}}$. From this 4.2 follows.

The case $y=0$ requires special care. Note that when $y=b=0$ then $L / \eta \leq a \leq x \leq \eta L$ so $f_{a, b}(l ; L)-C l_{L}^{\frac{1}{2}-\varepsilon}>1$ for all $l \leq L-K$, for a constant $K$ depending on $C$ and $\eta$. Also for a large enough constant $C^{\prime}$, we have for all $1 \leq k \leq K$ that

$$
f_{a 0}(l ; L)-C l_{L}^{\frac{1}{2}-\varepsilon} \geq f_{a 0}(l ; L-k)-C^{\prime} l_{L-k}^{\frac{1}{2}-\varepsilon} \text { for } l=1, \ldots, L-k-1 .
$$

Thus letting

$$
A_{k}=\left\{\sqrt{2 T_{l}} \geq f_{a, b}(l ; L-k)-C^{\prime} l_{L-k}^{\frac{1}{2}-\varepsilon} \text { for } l=1, \ldots, L-k-1\right\},
$$

we have

$$
\mathbb{Q}_{1}^{x^{2} / 2}(A) \leq \sum_{k=1}^{K} \mathbb{Q}_{1}^{x^{2} / 2}\left(A_{k} ; \sqrt{2 T_{L-k}}>1, T_{L-k+1} \in H_{0, \delta \vee 1}\right) .
$$

Using the Markov property we have

$$
\mathbb{Q}_{1}^{x^{2} / 2}\left(A_{k} ; \sqrt{2 T_{L-k}}>1, T_{L-k+1} \in H_{0, \delta \vee 1}\right) \leq \sum_{j=1}^{\infty} \mathbb{Q}_{1}^{x^{2} / 2}\left(A_{k} ; \sqrt{2 T_{L-k}} \in H_{j}\right) e^{-\frac{j^{2}}{2}} .
$$

Now applying 4.2 with $L-k$ in place of $L$ and using $L / \eta \leq a \leq x$ gives that this sum is at most

$$
\begin{aligned}
& \sum_{j=1}^{\infty} c \frac{(1+x-a)(1+j)}{(L-k)^{3 / 2}} \sqrt{\frac{x}{j}} e^{-\frac{(x-j)^{2}}{2(L-k)}} e^{-\frac{j^{2}}{2}} \\
& \leq c \frac{(1+x-a)}{(L-k)} e^{-\frac{x^{2}}{2(L-k)}} \leq c \frac{(1+x-a)}{L} e^{-\frac{x^{2}}{2 L}}
\end{aligned}
$$

for $1 \leq k \leq K$, so the desired upper bound follows also in the case $y=0$. In general, if $y \leq \sqrt{2}$ then $[y, y+\delta] \subseteq[0, \sqrt{2}+\delta]$ so the upper bound follows from the case $y=0$. This completes the proof of the upper bound (1.1).

We now prove the lower bound. Recall that $x \geq \sqrt{2}$ and we first assume that also $y \geq \sqrt{2}$. Let

$$
\text { Tube }_{C, \widetilde{C}}(l, L)=\left\{z \mid f_{a, b}(l ; L)+C l_{L}^{\frac{1}{2}-\varepsilon} \leq z \leq f_{x, y}(l ; L)+\widetilde{C} l_{L}^{\frac{1}{2}+\varepsilon}\right\}
$$

and set

$$
D=\left\{\sqrt{2 T_{l}} \in \text { Tube }_{C, \widetilde{C}}(l, L), l=1, \ldots, L-1, \sqrt{2 T_{L}} \in H_{y, \delta}\right\} .
$$


We claim that

$$
\text { Tube }_{C, \widetilde{C}}(l, L) \cap \sqrt{2 \mathbb{Z}^{+}} \neq \emptyset, \text { for all } l=1, \ldots, L-1,
$$

so that $D \neq \emptyset$. By the concavity of the square root, it suffices to show that $\widetilde{C} l_{L}^{\frac{1}{2}+\varepsilon}-C l_{L}^{\frac{1}{2}-\varepsilon} \geq \sqrt{2}$ for all $l=1, \ldots, L-1$. This is certainly true for $l=1$ or $l=L-1$ by our requirements that imply that $\widetilde{C} \geq C+\sqrt{2}$, and the general case follows since $f(z)=\widetilde{C} z^{\frac{1}{2}+\varepsilon}-C z^{\frac{1}{2}-\varepsilon}$ is monotone increasing in $z \geq 1$.

We now show that for $x, y \geq \sqrt{2}$

$$
\mathbb{Q}_{1}^{x^{2} / 2}(D) \geq c \frac{(x-a)(y-b)}{L} \sqrt{\frac{x / y}{L}} e^{-\frac{(x-y)^{2}}{2 L}} .
$$

To this end consider the event

$$
\begin{aligned}
E=\left\{\sqrt{2 \mathcal{L}_{1}} \in H_{x}, \sqrt{2 \mathcal{L}_{l}} \in{\text { Tube } C^{\prime}, C^{\prime \prime}}(l, L), l\right. & =2, \ldots, L-1 \\
\sqrt{2 \mathcal{L}_{L}} & \left.\in\left[y+\frac{\delta}{3}, y+\frac{2 \delta}{3}\right]\right\},
\end{aligned}
$$

where $C^{\prime}=C+\delta / 3$ and $C^{\prime \prime}=C+\delta$, so that $C^{\prime \prime}-C^{\prime} \geq 2 \delta / 3$.

We show below that for some $\widehat{c}>0$

$$
\mathbb{Q}_{1}^{x^{2} / 2}(D \cap E) \geq \widehat{c} \mathbb{Q}_{1}^{x^{2} / 2}(E),
$$

from which it will follow that

$$
\mathbb{Q}_{1}^{x^{2} / 2}(D) \geq \widehat{c} \mathbb{Q}_{1}^{x^{2} / 2}(E) .
$$

As before, using the Markov property

$$
\begin{aligned}
& \mathbb{Q}_{1}^{x^{2} / 2}(E)=\mathbb{Q}_{1}^{x^{2} / 2}\left(\sqrt{2 \mathcal{L}_{1}} \in H_{x}, \mathbb{Q}_{2}^{\mathcal{L}_{1}}\left(\sqrt{2 \mathcal{L}_{l}} \in \operatorname{Tube}_{C^{\prime}, C^{\prime \prime}}(l, L),\right.\right. \\
& \left.\left.l=2, \ldots, L-1 ; \sqrt{2 \mathcal{L}_{L}} \in\left[y+\frac{\delta}{3}, y+\frac{2 \delta}{3}\right]\right)\right) . \\
& \geq \mathbb{Q}_{1}^{x^{2} / 2}\left(\sqrt{2 \mathcal{L}_{1}} \in H_{x}\right) \inf _{z \in H_{x}} \mathbb{Q}_{2}^{z^{2} / 2}\left(\sqrt{2 \mathcal{L}_{l}} \in \operatorname{Tube}_{C^{\prime}, C^{\prime \prime}}(l, L),\right. \\
& \left.\quad l=2, \ldots, L-1 ; \sqrt{2 \mathcal{L}_{L}} \in\left[y+\frac{\delta}{3}, y+\frac{2 \delta}{3}\right]\right) \\
& =: \mathbb{Q}_{1}^{x^{2} / 2}\left(\sqrt{2 \mathcal{L}_{1}} \in H_{x}\right) \inf _{z \in H_{x}} K_{2}(z) .
\end{aligned}
$$


By 3.7 we have that $\mathbb{Q}_{1}^{x^{2} / 2}\left(\sqrt{2 \mathcal{L}_{1}} \in H_{x}\right) \geq c>0$ uniformly in all possible $x$. Once again, by $(3.5)$, if $Y=Y_{t}$ is a $\operatorname{Bes}^{0}(\sqrt{2 u})$ then

$$
\begin{aligned}
& K_{2}(z)=P\left(Y_{l} \in \text { Tube }_{C^{\prime}, C^{\prime \prime}}(l+1, L),\right. \\
& \left.\quad l=1, \ldots, L-1, Y_{L} \in\left[y+\frac{\delta}{3}, y+\frac{2 \delta}{3}\right] \mid Y_{0}=z\right),
\end{aligned}
$$

and using the Markov property and then $(2.9)$ (with $y$ replaced by $y+\delta / 3$ and with $\delta$ taken as $2 \delta / 3$ ), which requires $x, y \geq 1$ and $C^{\prime \prime}-C^{\prime} \geq 2 \delta / 3$, we obtain

$$
K_{2} \geq c \frac{(1+x-a)(1+y-b)}{L-1} \sqrt{\frac{x / y}{L-1}} e^{-\frac{(x-y)^{2}}{2(L-1)}},
$$

which because of our assumptions on $x, y, a, b$ is greater than or equal to the right-hand side of 4.20. Together with 4.22 this proves 4.20 for $x, y \geq \sqrt{2}$.

Assume now that $y \leq \sqrt{2}$, which by our assumption requires that $x \geq L / \eta$. The tube at $l=L-1$ contains some interval $[z, z+\bar{\delta}]$ with $z \geq \sqrt{2}$ such that $[z, z+\bar{\delta}] \cap \sqrt{2 \mathbb{Z}^{+}} \neq \emptyset$. We then apply 4.20 for the interval of length $L-1$ with

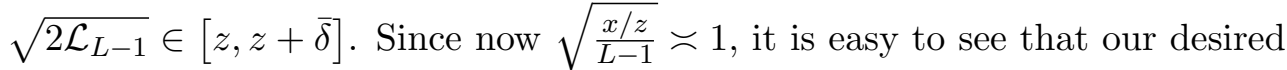
lower bound follows from the fact that there is some positive probability to go from $[z, z+\bar{\delta}]$ to some point in $[y, y+\delta] \cap \sqrt{2 \mathbb{Z}^{+}}$in a single step.

We now turn to the proof of 4.21. By part c) of Lemma 3.1.

$$
\begin{aligned}
\mathbb{Q}_{1}^{x^{2} / 2}(D \cap E)= & \mathbb{Q}_{1}^{x^{2} / 2}\left\{1_{E} \mathbb{Q}_{1}^{x^{2} / 2}\left(\sqrt{2 T_{1}} \in \text { Tube }_{C, \widetilde{C}}(1, L) \mid \mathcal{L}_{1}, \mathcal{L}_{2}\right)\right. \\
& \times\left(\prod_{l=2}^{L-1} \mathbb{Q}_{1}^{x^{2} / 2}\left(\sqrt{2 T_{l}} \in \text { Tube }_{C, \widetilde{C}}(l, L) \mid \mathcal{L}_{l}, \mathcal{L}_{l+1}\right)\right) \\
& \left.\times \mathbb{Q}_{1}^{x^{2} / 2}\left(\sqrt{2 T_{L}} \in H_{y} \mid \mathcal{L}_{L}\right)\right\} .
\end{aligned}
$$

Recall from 3.1 that the law of $\sqrt{2 T_{l}}$ depends only on the product $\mathcal{L}_{l} \mathcal{L}_{l+1}$. Note that

$$
\left|f_{x, y}(l+1 ; L)-f_{x, y}(l ; L)\right| \leq \eta
$$

with a similar bound for $f_{a, b}(l ; L)$. It follows that on the event $E$

$$
\left\{f_{a, b}(l ; L)+C^{\prime} l_{L}^{\frac{1}{2}-\varepsilon}-c^{\prime}\right\} \leq\left(4 \mathcal{L}_{l} \mathcal{L}_{l+1}\right)^{1 / 4} \leq\left\{f_{x, y}(l ; L)+C^{\prime \prime} l_{L}^{\frac{1}{2}+\varepsilon}+c^{\prime \prime}\right\},
$$

for $2 \leq l \leq L-1$ with $c^{\prime}=\eta+C^{\prime}$ and $c^{\prime \prime}=\eta+C^{\prime \prime}$. Thus we have that on $E$

$$
\left\{\left|\sqrt{2 T_{l}}-\left(4 \mathcal{L}_{l} \mathcal{L}_{l+1}\right)^{1 / 4}\right| \leq \frac{\delta}{10} l_{L}^{\frac{1}{2}-\varepsilon}\right\} \subseteq\left\{\sqrt{2 T_{l}} \in T u b e_{C, \widetilde{C}}(l, L)\right\},
$$


for $k \leq l \leq L-k$ for some fixed $k$ independent of $L$. Then by 3.16 we have

$$
\begin{aligned}
& 1_{E} \prod_{l=k}^{L-k} \mathbb{Q}_{1}^{x^{2} / 2}\left(\sqrt{2 T_{l}} \in \text { Tube }_{C, \widetilde{C}}(l, L) \mid \mathcal{L}_{l}, \mathcal{L}_{l+1}\right) \\
& \geq 1_{E} \prod_{l=k}^{L-k}\left(1-\exp \left(-c\left(\frac{\delta}{10}\right)^{2} l_{L}^{1-2 \varepsilon}\right)\right) \geq 1_{E} c_{*},
\end{aligned}
$$

for some $c_{*}>0$ independent of $L$.

Consider now $2 \leq l \leq k$ or $L-k \leq l \leq L-1$. By (4.28), on the event $E$ we have

$$
\left\{\sqrt{2 T_{l}} \in\left(4 \mathcal{L}_{l} \mathcal{L}_{l+1}\right)^{1 / 4}+[r, s]\right\} \subseteq\left\{\sqrt{2 T_{l}} \in \text { Tube }_{C, \widetilde{C}}(l, L)\right\},
$$

with

$$
[r, s]=\left[\eta+C^{\prime}, \eta+C^{\prime \prime}+\sqrt{2}\right] .
$$

Hence using (3.17) we see that for any

$$
1_{E} \mathbb{Q}_{1}^{x^{2} / 2}\left(\sqrt{2 T_{l}} \in \text { Tube }_{C, \widetilde{C}}(l, L) \mid \mathcal{L}_{l}, \mathcal{L}_{l+1}\right) \geq 1_{E} c_{l},
$$

for some $c_{l}>0$ which depends on $\eta, \delta$ and $C$.

In addition, on the event $E$ we have

$$
\begin{aligned}
\sqrt{a} \sqrt{f_{a, b}(2 ; L)+C^{\prime}} & \leq\left(4 \mathcal{L}_{1} \mathcal{L}_{2}\right)^{1 / 4} \\
& \leq \sqrt{x+1} \sqrt{f_{x, y}(2 ; L)+2 C^{\prime \prime}} .
\end{aligned}
$$

Thus, as in the last paragraph, we have by (3.17) that

$$
1_{E} \mathbb{Q}_{1}^{x^{2} / 2}\left(\sqrt{2 T_{1}} \in \text { Tube }_{C, \widetilde{C}}(1, L) \mid \mathcal{L}_{1}, \mathcal{L}_{2}\right) \geq 1_{E} c_{1}
$$

for some $c_{1}>0$ which depends on $\eta, \delta$ and $C$.

Finally, on the event $E$ we have

$$
\sqrt{2 \mathcal{L}_{L}} \in\left[y+\frac{\delta}{3}, y+\frac{2 \delta}{3}\right]
$$

For all $y \geq y_{0}$ sufficiently large, for any $v \in\left[y+\frac{\delta}{3}, y+\frac{2 \delta}{3}\right]$, we will have that $v+[0, \delta / 3] \cap \sqrt{2 \mathbb{N}} \neq \emptyset$. Then we have by 3.9 that for some for some $c_{L}>0$

$$
1_{E} \mathbb{Q}_{1}^{x^{2} / 2}\left(\sqrt{2 T_{L}} \in H_{y, \delta} \mid \mathcal{L}_{L}\right) \geq 1_{E} c_{L}
$$


On the other hand, we know from Lemma 3.1 a) that $T_{L}$ conditioned on $\mathcal{L}_{L}=u$ is Poisson with parameter $u$, and since by our assumption $H_{y, \delta} \cap \sqrt{2 \mathbb{Z}^{+}} \neq \emptyset$, it follows that for some possibly smaller $c_{L}>0$, (4.34) also holds if $y \leq y_{0}$.

This completes the proof of 4.21 with $\widehat{c}=c_{*} \prod_{l=1}^{k} c_{l} \prod_{j=L-k}^{L} c_{j}>0$, and hence of $(4.20)$.

\section{Tree cover time}

In this section we prove Theorem 1.3 about the tightness of the cover time of the regular tree. We begin this section by introducing notation. Recall that $\mathcal{T}_{L}$ denotes a rooted tree, with root $\rho$ to which one attaches a standard binary tree of depth $L$. Recall also that $\mathbb{P}$ is the probability of simple random walk on this tree starting from $\rho$. For a vertex $v \in \mathcal{T}_{L}$, we write $|v|$ for the tree distance of $v$ from the root $\rho$. We define level $l$ of the tree $(l=-1,0,1, \ldots, L)$ as those vertices at distance $l+1$ from $\rho$, and write $\mathbf{L}_{l}$ for the vertices of $\mathcal{T}_{L}$ at level $l$. For $y \in \mathbf{L}_{L}$, we let $[y]_{l}$ denote the ancestor of $y$ at level $l$, i.e. the unique vertex in $\mathbf{L}_{l}$ on the geodesic connecting $y$ and $\rho$; thus $[y]_{-1}$ is the root $\rho$.

Let $D_{n}$ be the time of the $n$-th return to the root $\rho$. For each $y \in \mathbf{L}_{L}$, define

$$
T_{l}^{y, n}=\# \text { of traversals of the edge }[y]_{l-1} \rightarrow[y]_{l} \text { up to time } D_{n} .
$$

The count $T_{l}^{y, n}$ is the edge local time of the directed edge $\left([y]_{l-1},[y]_{l}\right)$ accumlated during the first $n$ excursions from the root. Note that for $y \in \mathbf{L}_{L}$ we have

$$
\tau_{y}>D_{n} \text { if and only if } T_{L}^{y, n}=0 .
$$

When we observe the random walk on the tree only when it is on the path from $\rho$ to a leaf $y \neq \rho$, we obtain a one dimensional simple random walk on $\{-1,0,1, \ldots, L\}$. As such the following is simply a restatement of the classical fact that the directed edge local times of 1D simple random walk are a Markov process, and more precisely a critical Galton-Watson with geometric offspring distribution (this is the discrete equivalent of the second Ray-Knight theorem).

Lemma 5.1. For each $n$ and each leaf $y \in \mathbf{L}_{L}$ the process $\left(T_{l}^{y, n}\right)_{l=0, \ldots, L}$ has law $P_{n}$, i.e. is a Galton-Watson process with offspring distribution given by the geometric law with mean 1. 
Also note that

if $y, z$ are two different leaves in $\mathbf{L}_{L}$ whose paths from root to leaf overlap up to level $k$, then $T_{l}^{y, n}=T_{l}^{n, z}$ for $l \leq k$,

and conditionally on $T_{k}^{y, n}=T_{k}^{n, z}$ the processes $\left(T_{l}^{y, n}\right)_{l \geq k},\left(T_{l}^{n, z}\right)_{l \geq k}$ are independent. Thus, in fact, the $T_{l}^{y, n}$ are a collection of branching Galton-Watson process, like a branching random walk where the random walk is replaced by the Galton-Watson process. This analogy allows us to use methods from branching random walks to prove Theorem 1.3 .

Let $\left(T_{l}\right)_{l>0}$ denote a generic Galton-Watson process with this offspring distribution. A basic bound for the increments of $T_{l}$ is given by the following

$$
P_{n}\left(\left|\sqrt{2 T_{l}}-\sqrt{2 T_{0}}\right| \geq z\right) \leq c e^{-\frac{z^{2}}{2 l}}, \quad z \geq 0
$$

which is easily proved by computing the exponential moments of $T_{l}$ using the representation of $T_{l}$ as the sum of $T_{0}$ random variables, each being the product of a Bernoulli with parameter $\frac{1}{l}$ and a Geometric with parameter $\frac{1}{l}$, see [6, Lemma 4.6] (for $n=O\left(l^{2}\right)$ and $z=O(l)$ it is a special case of the more precise bound (1.9)).

Throughout this section we set

$$
\kappa=\kappa_{L}=\sqrt{2 \log 2}-\frac{\log L}{\sqrt{2 \log 2} .} .
$$

The main step in proving the upper bound 1.2 on the right tail is the following proposition which gives an upper bound in terms of excursion counts - later we relate the excursion counts to the actual clock of the random walk $X_{n}$ to obtain from this the upper bound of Theorem 1.3 .

Proposition 5.2. There is a constant $c$ such that for all $L \geq 1$ and $x \geq 1$ we have

$$
\mathbb{P}\left(\min _{y \in \mathbf{L}_{L}} T_{L}^{y, n}=0\right) \leq c x e^{-\kappa x},
$$

where $\sqrt{2 n}=\kappa L+x$.

Proof. The probability in $(5.4)$ is at most

$$
\begin{aligned}
& \underbrace{\mathbb{P}\left(\exists y \in \mathbf{L}_{L}, l \leq L-1 \text { s.t. } \sqrt{2 T_{l}^{y, n}}<\alpha(l)\right)}_{(\mathrm{I})}, \\
& +\underbrace{\mathbb{P}\left(\exists y \in \mathbf{L}_{L} \text { s.t. } \sqrt{2 T_{l}^{y, n}} \geq \alpha(l), l=1, \ldots, L-1, T_{L}^{y, n}=0\right)}_{\text {(II) }},
\end{aligned}
$$


where we have split the event according to whether or not the processes $T_{l}^{y, n}$ stay above the barrier

$$
\alpha(l)=\kappa(L-l)-l_{L}^{1 / 6}
$$

(as typically they should).

By a union bound over the levels of the tree we have,

$$
(\mathrm{I}) \leq \sum_{k=1}^{L-2} \mathbb{P}\left(\sqrt{2 T_{l}^{y, n}} \geq \alpha(l), l=1, \ldots, k, \sqrt{2 T_{k+1}^{y, n}}<\alpha(k+1)\right) .
$$

By Lemma 5.1, (5.2) and a union bound over the $2^{k}$ vertices in the $k$-th level of the tree the $k$-th summand is at most

$$
2^{k} P_{n}\left(\sqrt{2 T_{l}} \geq \alpha(l), l=1, \ldots, k, \sqrt{2 T_{k+1}}<\alpha(k+1)\right) .
$$

We condition on the height of the process at time $k$ to obtain an upper bound of

$$
\begin{gathered}
\sum_{j \geq 0} P_{n}\left(\sqrt{2 T_{l}} \geq \alpha(l), l=1, \ldots, k-1, \sqrt{2 T_{k}} \in H_{\alpha(k)+j}\right) \\
\times \sup _{z \in H_{\alpha(k)+j}} P_{\frac{z^{2}}{2}}\left(\sqrt{2 T_{1}} \leq \alpha(k+1)\right),
\end{gathered}
$$

for the probability in the summand in $(5.6)$. By (5.3),

$$
\sup _{z \in H_{\alpha(k)+j}} P_{\frac{z^{2}}{2}}\left(\sqrt{2 T_{1}} \leq \alpha(k+1)\right) \leq c e^{-j^{2} / 2} .
$$

To bound the first probability in (5.8), we use (1.1) with modified parameters, as follows. First, note that for any $\gamma<1$,

$$
l_{L}^{\gamma} \leq k_{L}^{\gamma}+l_{k}^{\gamma}
$$

Setting, for $l=0, \ldots, k, \hat{\alpha}(l)=f_{a, b}(l, L)-l_{k}^{1 / 6}$ with $a=\kappa L-k_{L}^{1 / 6}$ and $b=\kappa(L-k)-k_{L}^{1 / 6}$, we thus conclude that $\hat{\alpha}(l) \leq \alpha(l)$ with $\alpha(k)=\hat{\alpha}(k)$. Therefore,

$$
\begin{aligned}
& P_{n}\left(\sqrt{2 T_{l}} \geq \alpha(l), l=1, \ldots, k-1, \sqrt{2 T_{k}} \in H_{\alpha(k)+j}\right) \\
& \leq P_{n}\left(\sqrt{2 T_{l}} \geq \hat{\alpha}(l), l=1, \ldots, k-1, \sqrt{2 T_{k}} \in H_{\hat{\alpha}(k)+j}\right) .
\end{aligned}
$$

We now apply 1.1 with $L$ replaced by $k$, the $x$ of $\sqrt{1.1}$ replaced by $\sqrt{2 n}$, $y=\hat{\alpha}(k)+j$, and these values of $a, b$, and obtain that the right hand side in 5.10 is bounded by

$$
c \frac{\left(x+k_{L}^{1 / 6}\right)(j+1)}{k} e^{-\left(\kappa k+x-j+k_{L}^{1 / 6}\right)^{2} / 2 k} \leq c\left(x+k_{L}^{1 / 6}\right)(j+1) 2^{-k} e^{\kappa j-\kappa x-\kappa k_{L}^{1 / 6}} .
$$


Combining with (5.9) and summing over $j$ we get that $(5.8)$ is at most $c 2^{-k}\left(x+k_{L}^{1 / 6}\right) e^{-\kappa\left(x+k_{L}^{1 / 6}\right)}$. Thus from 5.6

$(\mathrm{I}) \leq c \sum_{k=1}^{L-2} 2^{k}\left(2^{-k}\left(x+k_{L}^{1 / 6}\right) e^{-\kappa x-\kappa k_{L}^{1 / 6}}\right)=c e^{-\kappa x} \sum_{k=1}^{L}\left(x+k_{L}^{1 / 6}\right) e^{-\kappa k_{L}^{1 / 6}} \leq c x e^{-\kappa x}$.

By Lemma 5.1 and a union bound over all leaves in $\mathbf{L}_{L}$ and (1.1) we have

$$
\text { (II) } \leq 2^{L} P_{n}\left(\sqrt{2 T_{l}} \geq \alpha(l), l=1, \ldots, L, T_{L}=0\right) \leq c x e^{-\kappa x},
$$

if $x \geq 1$. Together with (5.11) and (5.6), this proves (5.4).

To prove a lower bound in terms of excursion counts we will consider the processes

$$
T_{l}^{y, k, m}=\begin{gathered}
\# \text { of traversals from }[y]_{l-1} \text { to }[y]_{l} \text { during } \\
\text { the first } m \text { excursions from }[y]_{k} \text { to }[y]_{k-1}
\end{gathered}
$$

Like $\left(T_{l}^{y, n}\right)_{l \geq 0}$, the process $\left(T_{l+k}^{y, k, m}\right)_{l \geq 0}$ is a critical Galton-Watson process with geometric offspring, but with initial population $m$ i.e.

$$
\text { the law of }\left(T_{l+k}^{y, k, m}\right)_{l \geq 0} \text { under } \mathbb{P} \text { is } P_{m} \text {. }
$$

Also

$$
\left(T_{l+k}^{y, k, m}\right)_{l \geq 0} \text { and }\left(T_{l+k}^{z, k, m}\right)_{l \geq 0} \text { are independent if the }
$$

latest common ancestor of leaves $y \neq z$ in the tree is in or above level $k$.

Note further that

$$
\text { on the event } T_{k}^{y, n}=m \text { we have } T_{k+l}^{y, n}=T_{k+l}^{y, k, m} \text { for } l=0, \ldots L-k \text {. }
$$

The main step in proving the lower bound on the right tail (1.3) and the upper bound on the left tail (1.4) is the following result for the processes $T_{l}^{y, k, m}$. 
Lemma 5.3. There is a constant $c$ such that for all $x \geq 0$ and $k \geq 1$ we have

$$
\liminf _{L \rightarrow \infty} \mathbb{P}\left(\inf _{y \in \mathbf{L}_{L}} T_{L}^{y, k, m}=0\right) \geq \frac{2^{k}(1+x) e^{-x \sqrt{2 \log 2}}}{2^{k}(1+x) e^{-\sqrt{2 \log 2}+c}} .
$$

where

$$
\sqrt{2 m}=\bar{\kappa} \bar{L}+x, \text { for } \bar{L}=L-k, \text { and } \bar{\kappa}=\kappa_{\bar{L}}=\sqrt{2 \log 2}-\frac{1}{\sqrt{2 \log 2}} \frac{\log \bar{L}}{\bar{L}} .
$$

Proof. Consider the event

$$
I_{y}=\left\{\bar{\kappa}(\bar{L}-l)+\left(l_{\bar{L}}\right)^{1 / 4} \leq \sqrt{2 T_{k+l}^{y, k, m}} \text { for } l=1, \ldots, \bar{L}-1, T_{k+\bar{L}}^{y, k, m}=0\right\},
$$

that $\left(T_{l+k}^{y, k, m}\right)_{l \geq 0}$ stays above the barrier $\bar{\kappa}(\bar{L}-l)+\left(l_{\bar{L}}\right)^{1 / 4}$ and ends up at zero, and the counting random variable

$$
\mathcal{N}_{k}=\sum_{y \in \mathbf{L}_{L}} 1_{I_{y}} .
$$

If $\mathcal{N}_{k} \geq 1$ then $\min _{y \in \mathbf{L}_{L}} T_{L}^{y, k, m}=0$ so to prove 5.15 it suffices to show that for large enough $L$

$$
\mathbb{P}\left(\mathcal{N}_{k} \geq 1\right) \geq \frac{2^{k}(1+x) e^{-\bar{\kappa} x}}{2^{k}(1+x) e^{-\bar{\kappa} x}+c} .
$$

Using (1.1), (1.2) one has

$$
\mathbb{E}\left(\mathcal{N}_{k}\right) \asymp 2^{L} 2^{-\bar{L}}(1+x) e^{-\bar{\kappa} x}=2^{k}(1+x) e^{-\bar{\kappa} x},
$$

and by the Paley-Zygmund inequality

$$
\mathbb{P}\left(\mathcal{N}_{k} \geq 1\right) \geq \frac{\mathbb{E}\left(\mathcal{N}_{k}\right)^{2}}{\mathbb{E}\left(\mathcal{N}_{k}^{2}\right)}
$$

To prove (5.16) we estimate the second moment of $\mathcal{N}_{k}$. We have

$$
\begin{aligned}
\mathbb{E}\left(\mathcal{N}_{k}^{2}\right) & =\sum_{y, z} \mathbb{P}\left(I_{y} \cap I_{z}\right) \\
& =\sum_{y, z: \text { branch early }} \mathbb{P}\left(I_{y}\right) \mathbb{P}\left(I_{z}\right)+\sum_{y, z: \text { branch late }} \mathbb{P}\left(I_{y} \cap I_{z}\right),
\end{aligned}
$$

where the first sum is over pairs of leaves whose most recent common ancestor lie in level $k$ of the tree or above, so that the events $I_{y}$ and $I_{z}$ are independent, 
and the second sum is over all other pairs of leaves. The first sum is at most $2^{2 L} \mathbb{P}\left(I_{y}\right)^{2}=\mathbb{E}\left(\mathcal{N}_{k}\right)^{2}$. Thus (5.17) implies 5.16) once we have shown that for large enough $L$

$$
\sum_{y, z: \text { branch late }} \mathbb{P}\left(I_{y} \cap I_{z}\right) \leq c 2^{k}(1+x) e^{-\bar{\kappa} x}
$$

To bound this sum we sum over the possible level of the common ancestor of $y, z$ :

$$
\sum_{y, z: \text { branch late }} \mathbb{P}\left(I_{y} \cap I_{z}\right) \leq 2^{k} \sum_{j=0}^{\bar{L}} 2^{2 \bar{L}-j} p_{j},
$$

where $p_{j}=\mathbb{P}\left(I_{y} \cap I_{z}\right)$ for $y, z$ whose common ancestor is in level $k+j$ of the tree and we have used that there are at most $2^{k} 2^{2 \bar{L}-j}$ such pairs. Now by conditioning on the value of the processes at the point where they branch, we have

$$
p_{j} \leq \sum_{u \geq 0} q_{j}(u) r_{j}(u)^{2},
$$

where we sum over positive integers $u$ and where

$$
q_{j}(u)=P_{m}\left(\bar{\kappa}(\bar{L}-l) \leq \sqrt{2 T_{l}}, l=1, \ldots, j-1, \sqrt{2 T_{j}} \in H_{m_{u}}\right),
$$

and

$$
\begin{aligned}
& r_{j}(u) \\
& =\max _{z \in H_{m_{u}}} P_{\frac{z^{2}}{2}}\left(\bar{\kappa}(\bar{L}-j-l) \leq \sqrt{2 T_{l}}, l=1, \ldots, \bar{L}-j-1, T_{\bar{L}-j}=0\right),
\end{aligned}
$$

with

$$
m_{u}=\bar{\kappa}(\bar{L}-j)+j_{\bar{L}}^{1 / 4}+u
$$

By (1.1),

$$
q_{j}(u) \leq c \frac{1}{j}(1+x)\left(1+j_{\bar{L}}^{1 / 4}+u\right) e^{-\frac{\left(j \bar{\kappa}+x-j_{\bar{L}}^{1 / 4}-u\right)^{2}}{2 j}}
$$

and

$$
r_{j}(u) \leq c \frac{1}{\bar{L}-j}\left(1+j_{\bar{L}}^{1 / 4}+u\right) e^{-\frac{\left((\bar{L}-j) \bar{\kappa}+j_{\bar{L}}^{1 / 4}+u\right)^{2}}{2(\bar{L}-j)}}
$$


Since

$$
\begin{aligned}
& \frac{\left(j \bar{\kappa}+x-j_{\bar{L}}^{1 / 4}-u\right)^{2}}{2 j}+2 \frac{\left((\bar{L}-j) \bar{\kappa}+j_{\bar{L}}^{1 / 4}+u\right)^{2}}{2(\bar{L}-j)} \\
& \geq \frac{\bar{\kappa}^{2}}{2}(2 \bar{L}-j)+\bar{\kappa} x+c j_{\bar{L}}^{1 / 4}+c u,
\end{aligned}
$$

and $e^{-\frac{\bar{\kappa}^{2}}{2}(2 \bar{L}-j)}=\bar{L}^{\frac{2 \bar{L}-j}{\bar{L}}} 2^{-(2 \bar{L}-j)} \leq \bar{L}(\bar{L}-j) 2^{-2(\bar{L}-j)}$ we thus have from (5.20) that

$$
p_{j} \leq c 2^{-(2 \bar{L}-j)} \bar{L}(\bar{L}-j) \frac{1}{j(\bar{L}-j)^{2}}(1+x)\left(1+x+j_{\bar{L}}^{1 / 4}\right)^{3} e^{-\bar{\kappa} x-c j_{\bar{L}}^{1 / 4}}
$$

Thus the right-hand side of 5.19 is at most

$$
2^{k} \sum_{j=1}^{\bar{L}} \frac{\bar{L}}{j(\bar{L}-j)}(1+x)\left(1+j_{\bar{L}}^{1 / 4}\right)^{3} e^{-\bar{\kappa} x-c j_{\bar{L}}^{1 / 4}} .
$$

Since the sum is bounded by a constant for all $\bar{L}$ we get that

$$
\sum_{y, z: \text { branch late }} \mathbb{P}\left(I_{y} \cap I_{z}\right) \leq c 2^{k}(1+x) e^{-\bar{\kappa} x}
$$

which completes the proof of (5.18), and therefore also of (5.16) and $(5.22)$.

By simply setting $k=0$ and bounding $x e^{-x \sqrt{2 \log 2}}$ by a constant we obtain a lower bound on the right tail in terms of excursions. This will later lead to (1.3).

Corollary 5.4. For all $x \geq 1$,

$$
\liminf _{L \rightarrow \infty} \mathbb{P}\left(\inf _{y \in \mathbf{L}_{L}} T_{L}^{y, n}=0\right) \geq c x e^{-x \sqrt{2 \log 2}}
$$

where $\sqrt{2 n}=\kappa L+x$.

A lower bound for the cover time in terms of excursions, which will later lead to $(1.4)$ is given by the following proposition. 
Proposition 5.5. There is a constant $c$ such for all $x \geq 0$,

$$
\liminf _{L \rightarrow \infty} \mathbb{P}\left(\min _{y \in \mathbf{L}_{L}} T_{L}^{y, n}=0\right) \geq 1-c e^{-c x},
$$

where $\sqrt{2 n}=\kappa L-x$.

Proof. We will fix $k=k(x)$ below. From (5.14) it follows that

$$
T_{k}^{y, n} \leq m \text { and } T_{L-k}^{y, k, m}=0 \Longrightarrow T_{L}^{y, n}=0 .
$$

We consider the processes $\left(T_{k+l}^{y, k, m}\right)_{l=0, \ldots, \bar{L}}$ where $\bar{L}=L-k$. Using 5.3 we have for any $k, a, L \geq 1$ that

$$
\mathbb{P}\left(\max _{y \in \mathbf{L}_{L}} \sqrt{2 T_{k}^{y, n}}>(\kappa L-x)+\kappa k+a\right) \leq 2^{k} e^{-\frac{(\kappa k+a)^{2}}{2 k}} \leq c L^{k / L} e^{-\kappa a},
$$

by a union bound over the $2^{k}$ vertices in level $k$ of the tree, which is justified by 5.2. Setting $k=c x$ and $a=x-2 \kappa k-\frac{k}{c} \frac{\log L}{L}$ one has $\bar{\kappa} \bar{L} \geq \kappa L-x+\kappa k+a$ and $a \geq c x$ for small enough $c$ and large enough $L$, so that

$$
\limsup _{L \rightarrow \infty} \mathbb{P}\left(\max _{y \in \mathbf{L}_{L}} \sqrt{2 T_{k}^{y, n}}>\bar{\kappa} \bar{L}\right) \leq c e^{-c x} .
$$

Setting $\sqrt{2 m}=\bar{\kappa} \bar{L}$ and using Lemma 5.3 with 0 in place of $x$ it follows that

$$
\liminf _{L \rightarrow \infty} \mathbb{P}\left(\inf _{y \in \mathbf{L}_{L}} T_{L}^{y, k, m}=0\right) \geq \frac{2^{k}}{2^{k}+c} \geq 1-c 2^{-k} .
$$

Together with (5.24) this implies

$$
\begin{aligned}
& \liminf _{L \rightarrow \infty} \mathbb{P}\left(\left\{\forall y \in \mathbf{L}_{L} T_{k}^{y, k} \leq m\right\} \cap\left\{\exists y \in \mathbf{L}_{L} \text { s.t. } T_{L}^{y, k, m}=0\right\}\right) \\
& \geq 1-c 2^{-k}-c e^{-c x}
\end{aligned}
$$

which implies (5.22), because of (5.23) and since $k=c x$.

We now relate excursion time to real time to derive Theorem 1.3 from Proposition 5.2 and Proposition 5.5. Note that

$$
D_{n}=S_{1}+S_{2}+\ldots+S_{n},
$$


where $S_{i}$ is the length of the $i$-th excursion from the root. By the strong Markov property the $S_{i}, i \geq 1$, are iid. Elementary 1D random walk computations show that $\mathbb{E}\left(S_{i}\right)=2^{L+2}-2$ and by Khasminskii's lemma (a consequence of Kac's moment formula, see (6) [21] we have $\mathbb{E}\left(S_{i}^{k}\right) \leq k ! \mathbb{E}\left(S_{i}\right)^{k}$. Thus $\mathbb{E}\left(S_{i}^{2}\right) \leq c 2^{2 L}<\infty$, so that by the central limit theorem $\frac{D_{n}-n \mathbb{E}\left(S_{1}\right)}{\sqrt{n} \sqrt{\operatorname{Var}\left(S_{1}\right)}}$ converges to a normal distribution as $n \rightarrow \infty$. Also $\mathbb{E}\left(S_{i}^{3}\right) \leq c 2^{3 L} \leq c\left(\sqrt{\operatorname{Var}\left(S_{i}\right)}\right)^{2}$ (it is easily seen that $\operatorname{Var}\left(S_{i}\right) \geq c 2^{2 L}$ ), so that by the Berry-Essen theorem in addition

$$
\sup _{x \in \mathbb{R}}\left|\mathbb{P}\left(\frac{D_{n}-n \mathbb{E}\left(S_{1}\right)}{\sqrt{n} \sqrt{\operatorname{Var}\left(S_{1}\right)}} \leq x\right)-\Phi(x)\right| \leq \frac{c}{\sqrt{n}},
$$

uniformly in $L$. We now prove the estimate Theorem 1.3 for the cover time.

Proof of Theorem 1.3. For the upper bound, let

$$
\sqrt{2 n}=\sqrt{2 \log 2} L-\frac{1}{\sqrt{2 \log 2}} \log L+\frac{x}{2} .
$$

Because of (5.1) we have

$$
\begin{aligned}
& \mathbb{P}\left(C_{L}>2^{L+1}\left(\sqrt{2 \log 2} L-\frac{1}{\sqrt{2 \log 2}} \log L+x\right)^{2}\right) \\
& \leq \mathbb{P}\left(D_{n}>2^{L+1}\left(\sqrt{2 \log 2} L-\frac{1}{\sqrt{2 \log 2}} \log L+x\right)^{2}\right)+\mathbb{P}\left(\min _{y \in \mathbf{L}_{L}} T_{L}^{y, n}=0\right) .
\end{aligned}
$$

Since

$$
\begin{aligned}
& 2^{L+1}\left(\sqrt{2 \log 2} L-\frac{1}{\sqrt{2 \log 2}} \log L+x\right)^{2} \\
& \quad=2^{L+2} L((\log 2) L-\log L+\sqrt{2 \log 2} x+o(1)),
\end{aligned}
$$

and

$$
n=L\left((\log 2) L-\log L+\sqrt{2 \log 2} \frac{x}{2}+o(1)\right),
$$

we have from $(5.26)$ that

$$
\mathbb{P}\left(D_{n}>2^{L+1}\left(\sqrt{2 \log 2} L-\frac{1}{\sqrt{2 \log 2}} \log L+\sqrt{2 \log 2} x\right)^{2}\right) \leq c e^{-c x^{2}}+\frac{c}{\sqrt{n}},
$$

and combining this with Proposition 5.2 we get the claim (1.2) from (5.27). Since the right-hand side tends to zero as $x \rightarrow \infty$ this proves the upper bound 
on the right tail $(1.2)$. The lower bound $(1.3)$ on the right tail follows similarly from (5.21) and (5.26).

For the bound (1.4) on the left tail let

$$
\sqrt{2 n}=\sqrt{2 \log 2} L-\frac{1}{\sqrt{2 \log 2}} \log L-\frac{x}{2} .
$$

We have that

$$
\begin{aligned}
& \mathbb{P}\left(C_{L} \leq 2^{L+1}\left(\sqrt{2 \log 2} L-\frac{1}{\sqrt{2 \log 2}} \log L-x\right)^{2}\right) \\
& \leq \mathbb{P}\left(D_{n} \leq 2^{L+1}\left(\sqrt{2 \log 2} L-\frac{1}{\sqrt{2 \log 2}} \log L-x\right)^{2}\right)+\mathbb{P}\left(\min _{y \in \mathbf{L}_{L}} T_{L}^{y, n}>0\right) .
\end{aligned}
$$

Similarily to above $(5.26)$ implies that is at most $c e^{-x^{2}}$, and the second term is at most $c e^{-c x}$ by Proposition 5.5, which implies (1.4.

\section{References}

[1] Y. Abe. Extremes of local times for simple random walks on symmetric trees arXiv:1603.09047, 2016.

[2] L. Addario-Berry and B. Reed. Minima in branching random walks. Annals Probab., 37(3):1044-1079, 2009.

[3] E. Aïdékon. Convergence in law of the minimum of a branching random walk. Annals Probab., 41(3A):1362-1426, 2013.

[4] D. J. Aldous. Random walk covering of some special trees. J. Math. Anal. Appl., 157:271-283, 1991.

[5] L.-P. Arguin, A. Bovier, and N. Kistler. Genealogy of extremal particles of branching Brownian motion. Comm. Pure Appl. Math., 64(12):1647$1676,2011$.

[6] D. Belius and N. Kistler. The subleading order of two dimensional cover times. Probab. Theory Related Fields, 167(1):461-552, 2017.

[7] M. Bramson. Convergence of solutions of the Kolmogorov equation to traveling waves. Memoirs of the American Mathematical Society, 44(285):1-190, 1983. 
[8] M. Bramson, J. Ding and O. Zeitouni. Convergence in law of the maximum of nonlattice branching random walk. Annales Inst. H. Poincare 52:1897-1924, 2016.

[9] M. Bramson and O. Zeitouni. Tightness for a family of recursion equations. Annals Probab. 37:615-653, 2009.

[10] P. Carr and M. Schröder. Bessel processes, the integral of geometric Brownian motion, and Asian options. Teor. Veroyatnost. i Primenen., 48(3):503-533, 2003.

[11] A. Dembo, Y. Peres, J. Rosen and O. Zeitouni. Cover times for Brownian motion and random walks in two dimensions. Ann. Math. 160:433-464, 2004 .

[12] J. Ding. Asymptotics of cover times via Gaussian free fields: Bounded degree graphs and general trees. Annals Probab. 42:464-496, 2014.

[13] J. Ding, J. Lee and Y. Peres. Cover times, blanket times, and majorizing measures. Annals Math. 175:1409-1471, 2012.

[14] J. Ding and O. Zeitouni. A sharp estimate for cover times on binary trees. Stochastic Process. Appl. 122 2117-2133, 2012.

[15] W. Feller. An Introduction to Probability Theory and its Applications, Vol. II. New York: Wiley, 1971.

[16] I. Gradshteyn and I. Ryzhik, Table of Integrals, Series and Products, Academic Press, Oxford, (1980).

[17] J. Pitman and M. Yor. A decomposition of Bessel bridges. Z. Wahrsch. Verw. Gebiete, 59(4):425-457, 1982.

[18] D. Revuz and M. Yor. Continuous martingales and Brownian motion, volume 293. Springer-Verlag, Berlin, third edition, 1999.

[19] L. Yuan and J. D. Kalbfleisch, On the Bessel distribution and related problems. Annals of the Institute of Statistical Mathematics, 52:438-447, 2000.

[20] J. Pitman and M. Yor. Bessel processes and infinitely divisible laws. In 'Stochastic Integrals', ed. D. Williams. LNM 851. p. 285-370, 1981. Springer, NY. 
[21] P. J. Fitzsimmons and J. Pitman. Kac's moment formula and the Feynman-Kac formula for additive functionals of a Markov process. Stochastic Process. Appl. 79(1):117-134, 1999.

David Belius

Institute of Mathematics

University of Zürich

CH-8057 Zürich, Switzerland

david.belius@cantab.net

Jay Rosen

Department of Mathematics

College of Staten Island, CUNY

Staten Island, NY 10314

jrosen30@optimum.net

Ofer Zeitouni

Faculty of Mathematics, Weitzmann Institute and

Courant Institute, NYU

Rehovot 76100, Israel and NYC, NY 10012

ofer.zeitouni@weizmann.ac.il 